\title{
Eddy Influences on Hadley Circulations: Simulations with an Idealized GCM
}

\author{
Christopher C. WAlkeR* AND TAPio Schneider \\ California Institute of Technology, Pasadena, California
}

(Manuscript received 10 November 2005, in final form 19 April 2006)

\begin{abstract}
An idealized GCM is used to investigate how the strength and meridional extent of the Hadley circulation depend on the planet radius, rotation rate, and thermal driving. Over wide parameter ranges, the strength and meridional extent of the Hadley circulation display clear scaling relations with regime transitions, which are not predicted by existing theories of axisymmetric Hadley circulations. For example, the scaling of the strength as a function of the radiative-equilibrium equator-to-pole temperature contrast exhibits a regime transition corresponding to a regime transition in scaling laws of baroclinic eddy fluxes. The scaling of the strength of the cross-equatorial Hadley cell as a function of the latitude of maximum radiative-equilibrium temperature exhibits a regime transition from a regime in which eddy momentum fluxes strongly influence the strength to a regime in which the influence of eddy momentum fluxes is weak.

Over a wide range of flow parameters, albeit not always, the Hadley circulation strength is directly related to the eddy momentum flux divergence at the latitude of the streamfunction extremum. Simulations with hemispherically symmetric thermal driving span circulations with local Rossby numbers in the horizontal upper branch of the Hadley circulation between 0.1 and 0.8 , indicating that neither nonlinear nearly inviscid theories, valid for Ro $\rightarrow 1$, nor linear theories, valid for Ro $\rightarrow 0$, of axisymmetric Hadley circulations can be expected to be generally adequate. Nonlinear theories of axisymmetric Hadley circulations may account for aspects of the circulation when the maximum radiative-equilibrium temperature is displaced sufficiently far away from the equator, which results in cross-equatorial Hadley cells with nearly angular momentumconserving upper branches.

The dependence of the Hadley circulation on eddy fluxes, which are themselves dependent on extratropical circulation characteristics such as meridional temperature gradients, suggests that tropical circulations depend on the extratropical climate.
\end{abstract}

\section{Introduction}

The poleward mass flux along isentropes in the extratropical upper troposphere is associated with baroclinic eddy fluxes of potential vorticity; the equatorward mass flux along isentropes in the extratropical lower troposphere is associated with baroclinic eddy fluxes of surface potential temperature and potential vorticity (Held and Schneider 1999; Koh and Plumb 2004; Schneider 2005). In the subtropics, these eddy mass fluxes connect to the mass fluxes of the tropical Hadley circulation. Since a substantial fraction of the

* Current affiliation: Department of Earth and Planetary Science, Harvard University, Cambridge, Massachusetts.

Corresponding author address: Tapio Schneider, California Institute of Technology, Mail Code 100-23, 1200 E. California Blvd., Pasadena, CA 91125.

E-mail: tapio@caltech.edu isentropic mass flux of the Hadley circulation does not recirculate within the Tropics but connects continuously to the extratropical isentropic mass fluxes to form overturning circulations that span hemispheres (Fig. 1), it is possible that the baroclinic eddies that effect the extratropical mass fluxes influence the Hadley circulation. Indeed, the Hadley circulation in axisymmetric models with hemispherically symmetric thermal driving is weaker by approximately $50 \%-75 \%$ (depending on the specifics of the model) than in eddy-permitting models, making eddy influences on the simulated Hadley circulations manifest (Schneider 1984; Kim and Lee 2001; Becker et al. 1997; Walker and Schneider 2005).

As the extratropical mass fluxes are associated with eddy fluxes of potential vorticity, which, in turn, are a combination of eddy fluxes of heat and momentum, the mechanisms through which baroclinic eddies may influence the Hadley circulation are in question. In existing theories of idealized axisymmetric Hadley circulations, 

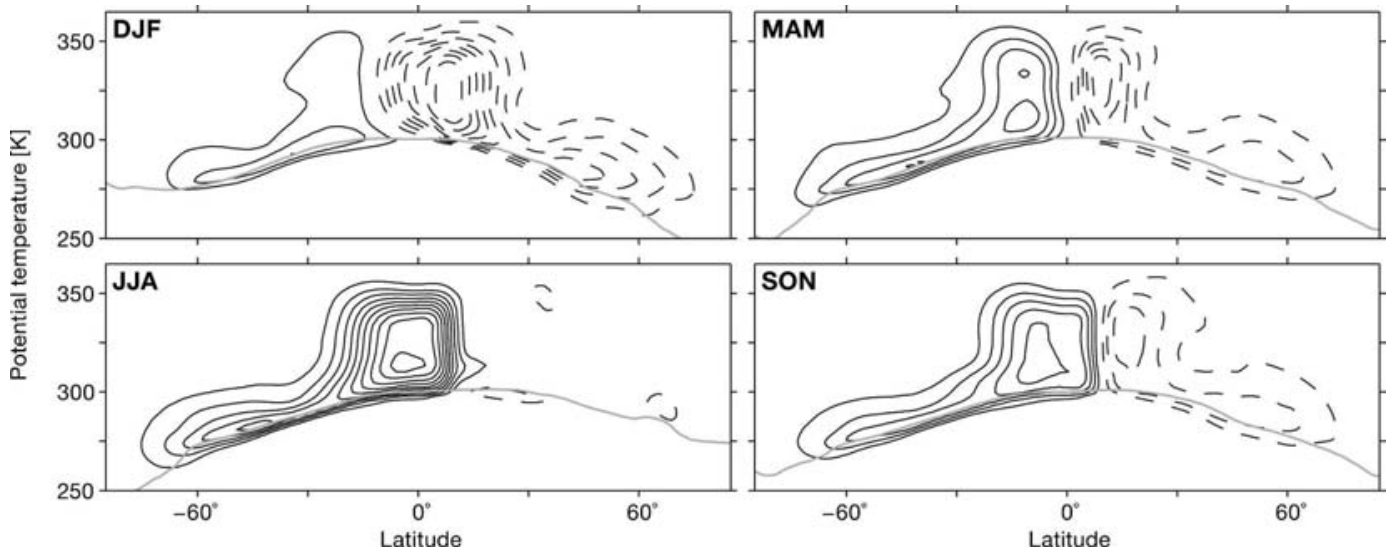

FIG. 1. Isentropic mass flux streamfunctions for December-February (DJF), March-May (MAM), June-August (JJA), and September-November (SON). Gray lines show median surface potential temperature. Contour interval is $25 \times 10^{9} \mathrm{~kg} \mathrm{~s}^{-1}$, with dashed (negative) contours indicating clockwise motion and solid (positive) contours indicating counterclockwise motion. Computed from reanalysis data for the years 1980-2001 provided by the European Centre for Medium-Range Weather Forecasts (Kållberg et al. 2004; Uppala et al. 2005).

such as the viscous linear theories of Dickinson (1971) and Schneider and Lindzen $(1976,1977)$ and the nearly inviscid nonlinear theories of Schneider (1977) and Held and Hou (1980), eddy heat fluxes can be incorporated by modifying the diabatic heating that drives the axisymmetric circulations (cf. Schneider 1984). Aside from the unsolved question of how eddy heat fluxes depend on the mean state that is determined, in part, by the Hadley circulation itself, incorporating eddy heat fluxes thus presents no fundamental problem to the existing axisymmetric Hadley circulation theories. Incorporating eddy momentum fluxes is more difficult.

In the temporal and zonal mean, the zonal momentum balance in the upper troposphere is primarily a balance between meridional advection of angular momentum and divergence of eddy momentum fluxes; that is,

$$
(f+\bar{\zeta}) \bar{v}=f(1-\mathrm{Ro}) \bar{v} \approx S
$$

where $v$ is the meridional velocity, $f$ the planetary vorticity (Coriolis parameter), $\zeta$ the relative vorticity, Ro $=-\bar{\zeta} / f$ is a local Rossby number, and $S$ is the eddy momentum flux divergence. The absolute vorticity $f+\bar{\zeta}=-\left(a^{2} \cos \phi\right)^{-1} \partial_{\phi} \bar{m}$ is proportional to the meridional gradient of the absolute angular momentum per unit mass, $\bar{m}=(\Omega a \cos \phi+\bar{u}) a \cos \phi$ (with symbols having their usual meaning). Overbars denote a temporal and zonal mean along isobaric surfaces. In the zonal momentum balance (1), we have neglected the vertical advection of zonal momentum by the mean circulation, which is generally small in the Tropics of Earth's atmosphere and vanishes near the latitudes of extrema of the Eulerian meridional streamfunction, where mean vertical velocities vanish. In the extratropics, the Rossby number is small, so the mean circulation (here and henceforth understood as the Eulerian mean circulation) is tightly coupled to the eddy momentum flux divergence; any change in $S$ is met by a proportional response in $\bar{v}$. In the Tropics, the low Rossby number approximation may no longer be appropriate, in which case the mean circulation may no longer be directly tied to the eddy momentum flux divergence; a change in $S$ can be met by a change in either $\bar{v}$ or in the absolute vorticity $f+\bar{\zeta}$ (i.e., a change in Rossby number Ro).

Linear theories of axisymmetric Hadley circulations consider the limit Ro $\rightarrow 0$, in which the eddy momentum flux divergence, often modeled as vertical momentum diffusion representing small-scale vertical mixing, directly influences the mean circulation. In the limit Ro $\rightarrow 0$, angular momentum contours are vertical lines, and eddy momentum fluxes make it possible that streamlines of the mean circulation cross angular momentum contours. Nearly inviscid nonlinear theories of axisymmetric Hadley circulations consider the limit of vanishing eddy momentum flux divergence in the upper troposphere. In this limit, angular momentum is conserved following the mean upper-tropospheric circulation, and, in a steady state, angular momentum contours and upper-tropospheric streamlines coincide. Where streamlines are horizontal, the nearly inviscid limit implies a vanishing meridional angular momentum gradient, or vanishing absolute vorticity, and thus Ro $\rightarrow 1$. In the nearly inviscid limit, the mean circulation decouples from the eddy momentum flux divergence. 

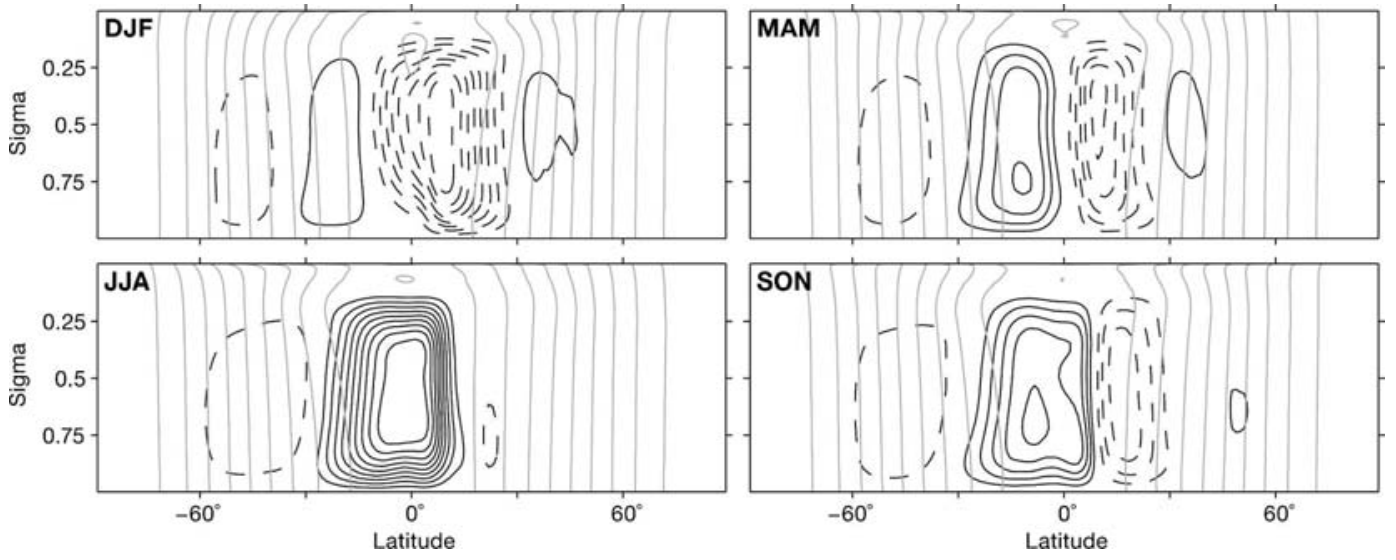

FIG. 2. Mass flux streamfunctions (black contours) and angular momentum (gray contours) for DJF, MAM, JJA, and SON. Streamfunction contour interval is $25 \times 10^{9} \mathrm{~kg} \mathrm{~s}^{-1}$; dashed and solid contours have the same meaning as in Fig. 1. Contour interval for angular momentum is $0.1 \Omega a^{2}$, with values decreasing monotonically away from the equator. Computed from the same reanalysis data as Fig. 1.

The existing theories of Hadley circulations thus neglect nonlinear momentum fluxes in the upper branches associated either with the mean circulation $(\mathrm{Ro} \rightarrow 0)$ or with eddies $(\mathrm{Ro} \rightarrow 1)$. We do not have theories for the case of intermediate Rossby numbers, in which nonlinear momentum fluxes associated both with eddies and with the mean circulation are important in the upper branches of the circulations.

There is evidence that nonlinear momentum fluxes associated both with eddies and with the mean circulation are important for Earth's Hadley circulation, with the relative importance of the eddy and mean momentum fluxes shifting over the course of the seasonal cycle. Figure 2 shows the mass flux streamfunction and contours of absolute angular momentum $\bar{m}$. In the winter hemispheres of the solstice seasons, and in both hemispheres of the equinox seasons, angular momentum contours in the tropical upper troposphere are displaced from the vertical, indicating nonlinear momentum fluxes associated with the mean Hadley circulation. Local Rossby numbers in the horizontal upper branches of the circulations, near the latitudes of the streamfunction extrema, are $0.2 \lesssim$ Ro $\lesssim 0.5$ in the equinox seasons and $\mathrm{Ro} \approx 0.5$ in the cross-equatorial winter Hadley cell in the December-February (DJF) season. Local Rossby numbers are poorly defined near the latitude of the streamfunction extremum of the crossequatorial winter Hadley cell in the June-August (JJA) season because the extremum is near the equator. In the summer hemispheres of the solstice seasons, angular momentum contours are less strongly displaced from the vertical, indicating weaker nonlinear momentum fluxes associated with the weaker mean Hadley circulation. Upper-tropospheric streamlines cross angu- lar momentum contours, indicating that angular momentum is not conserved in the upper branches of the summer Hadley cells. Local Rossby numbers in the upper branches of the summer Hadley cells are Ro $\lesssim 0.2$. The displacement of angular momentum contours from the vertical in winter and in the equinox seasons suggests that theories for the limit Ro $\rightarrow 0$ may not generally be adequate. However, theories for the limit Ro $\rightarrow 1$ in the upper branch are likewise not generally adequate, in particular not for the summer Hadley cells.

As the Hadley circulation does not generally conserve angular momentum in its upper branch, inferences of its response to changes in external parameters that are based on nearly inviscid axisymmetric theories may not be valid. Indeed, one such inference, that the strength of the annually averaged Hadley circulation is much larger than that of the Hadley circulation driven by annually averaged or equinoctial heating (Lindzen and Hou 1988), is not realized in Earth's atmosphere (Dima and Wallace 2003), in idealized eddy-permitting models, or in axisymmetric models with small vertical diffusion of potential temperature and momentum (Walker and Schneider 2005).

To provide guidance for and to test theories of how the Hadley circulation may respond to changes in climate, here we explore how the strength and meridional extent of the Hadley circulation behave in simulations with an idealized GCM in which moist processes are not explicitly taken into account. We vary external parameters such as the radiative-equilibrium surface temperature gradient and the planetary rotation rate over wide ranges to identify scaling laws for the strength and extent of the Hadley circulation. We compare these scaling laws with those predicted by existing theories, 
including nearly inviscid axisymmetric theories, which represent one important limit of atmospheric circulations, although they were not necessarily intended to account for nonaxisymmetric circulations.

In section 2, we review the scaling laws for the strength and meridional extent of the Hadley circulation as given by the nearly inviscid axisymmetric theory of Held and Hou (1980). In section 3, we describe the idealized GCM and parameter settings. In sections 4 and 5, we show how the strength and extent of the Hadley circulation depend on selected external parameters, in simulations with hemispherically symmetric thermal driving and with maximum heating displaced off the equator, respectively. In section 6 , we summarize the conclusions.

\section{Held-Hou model of the Hadley circulation}

Building on the work of Schneider (1977), Held and Hou (1980, hereafter HH) offered a theory of the axisymmetric circulation that results in the nearly inviscid limit of a model in which the thermal driving is given by Newtonian relaxation of temperatures toward a radiative-convective equilibrium state with $\cos ^{2} \phi$ dependence of temperature on latitude $\phi$. The theory is based on the assumptions that (i) the poleward flow in the upper branch of the Hadley circulation is approximately angular momentum-conserving, and (ii) the Hadley circulation is energetically closed, so that diabatic heating in the ascent regions is balanced by diabatic cooling in the descent regions. In the small-angle approximation (adequate in the Tropics), the theory leads to the following scaling relations for the Hadley circulation extent (latitude $\phi_{H}$ of poleward boundary) and strength (maximum absolute value $\Psi_{\max }$ of mass flux streamfunction):

$$
\begin{aligned}
\phi_{H} & \sim\left(R \frac{\Delta_{h}}{\theta_{0}}\right)^{1 / 2}, \\
\Psi_{\max } & \sim \rho_{0} \frac{a^{2} H_{t}}{\tau}\left(R \frac{\Delta_{h}}{\theta_{0}}\right)^{3 / 2} \frac{\Delta_{h}}{\Delta_{v}},
\end{aligned}
$$

where $R=g H_{t} /\left(\Omega^{2} a^{2}\right), H_{t}$ is the height of the tropopause, $\tau$ is a thermal relaxation time, taken to be constant, and $\rho_{0}$ is a reference density. The parameters $\theta_{0}$ and $\Delta_{h}$ are a global-mean potential temperature and the equator-to-pole radiative-convective equilibrium potential temperature difference vertically averaged over the troposphere. The parameter $\Delta_{v}$ is the gross stability, the effective potential temperature difference between the upper and lower branches of the Hadley circulation, a stability parameter that $\mathrm{HH}$ assumed to be externally imposed (e.g., as the tropopause-to-surface potential temperature difference in radiative-convective equilib- rium). However, the scaling relation (2b) for the strength of the circulation remains valid with the gross stability $\Delta_{v}$ in dynamical equilibrium if the gross stability cannot be viewed as externally imposed. The tropopause height in the scaling relations (2) is to be understood as a height scale of the Hadley circulation, which may be smaller than, for example, the height of the coldest point in an atmospheric column.

Different functional forms for the latitude dependence of the radiative-convective equilibrium temperature lead to different scaling relations for the Hadley circulation extent and strength. In the HH model with a $\cos ^{2} \phi$ dependence of vertically averaged radiativeconvective equilibrium potential temperatures on latitude, the Hadley circulation extent and strength, in the small-angle approximation according to relations (2), scale as $\left(H_{t}^{\prime} \Delta_{h}^{\prime}\right)^{1 / 2}$ and $\left(H_{t}^{\prime} \Delta_{h}^{\prime}\right)^{5 / 2}$, where $H_{t}^{\prime}=H_{t} / H_{0}$ is a nondimensional tropopause height and $\Delta_{h}^{\prime}=\Delta_{h} / \theta_{0}$ is the nondimensional potential temperature contrast in radiative-convective equilibrium. The exponents in these power laws, in the Boussinesq approximation underlying them, depend on the exponent of the leadingorder term in a small-angle expansion of the latitude dependence of the vertically averaged radiativeconvective equilibrium potential temperature. We will consider circulations with thermal driving not identical to that considered by $\mathrm{HH}$ but close to it in that the leading-order term in a small-angle expansion of the latitude dependence of the vertically averaged radiative-convective equilibrium potential temperature is likewise quadratic. We will discuss the extent to which the differences with the $\mathrm{HH}$ model may affect our simulation results and will mention the expected behavior according to HH's theory for those of our simulation results for which the small-angle approximation underlying the scaling relations (2) is not adequate.

\section{Model and parameters}

The idealized GCM and many of the simulations are the same as those described by Schneider and Walker (2006); only a brief overview is given here. The GCM is a hydrostatic, primitive equation model that employs the spectral transform method to integrate the equations in vorticity/divergence form (Bourke 1974). The spherical model surface is spatially uniform and thermally insulating, without topography and with a constant roughness length of $5 \mathrm{~cm}$. Radiative forcing is represented by Newtonian relaxation of temperatures toward a radiative-equilibrium state of a semigray atmosphere, which is statically unstable in the lower troposphere. As in the simulations of Schneider (2004), the thermal relaxation time near the surface (at $\sigma$ levels below $\sigma=0.85$ ) varies in most simulations from 7 days 
at the equator to 50 days at the poles, with a $\cos ^{8} \phi$ dependence of its inverse on latitude. In the interior atmosphere (at $\sigma$ levels above $\sigma=0.85$ ), the thermal relaxation time is constant and equal to 50 days. For a more direct comparison with nearly inviscid axisymmetric theories in which the thermal relaxation time is taken to be constant, we also conducted series of simulations in which the thermal relaxation time is constant and equal to 50 days throughout the atmosphere.

Phase changes of water are not explicitly taken into account in the model. However, if an atmospheric column is less stable than a column with temperature lapse rate $\gamma \Gamma_{d}$, where $\gamma$ is a rescaling parameter and $\Gamma_{d}=g / c_{p}$ is the dry adiabatic lapse rate, a quasi-equilibrium convection scheme relaxes temperatures toward a profile with lapse rate $\gamma \Gamma_{d}$. For $\gamma=1$, the convection scheme is a dry convection scheme; for $\gamma<1$, the convection scheme mimics the stabilizing effect of latent heat release in moist convection, with the implied latent heat release increasing with decreasing $\gamma$. Hyperdiffusion $\left(\nabla^{8}\right)$ in the vorticity, divergence, and temperature equations damps the smallest resolved scales on a time scale of $12 \mathrm{~h}$. Aside from the Newtonian relaxation of temperatures, hyperdiffusion is the only dissipative process above the planetary boundary layer, whose height is fixed at $2.5 \mathrm{~km}$.

We used four zonally symmetric radiative-equilibrium states: a normative state and three variants. The normative state is identical to that in Schneider and Walker (2006). It is symmetric about the equator and, like the radiative-convective equilibrium state of the HH model, has a $\Delta_{h} \cos ^{2} \phi$ surface temperature dependence on latitude, where the parameter $\Delta_{h}$ sets the equator-to-pole surface temperature difference. The radiative-equilibrium surface temperature at the poles is $260 \mathrm{~K}$ for all values of $\Delta_{h}$, which results in an increase of the mean radiative-equilibrium surface temperature $T_{0}=260 \mathrm{~K}+2 / 3 \Delta_{h}$ with $\Delta_{h}$. The increase of mean surface temperature leads to an increase of mean tropopause height with $\Delta_{h}$. (Increasing the mean surface temperature with $\Delta_{h}$ by holding the polar temperature fixed was necessary to ensure physical realizability of the radiative-equilibrium state for larger $\Delta_{h}$, for which otherwise the radiative-equilibrium surface temperature at the poles would have become smaller than a prescribed constant temperature at the top of the atmosphere, hence implying a negative optical thickness of the atmosphere.) The simulations with the normative radiative-equilibrium state and with spatially varying thermal relaxation time analyzed here are the same as those whose extratropical macroturbulence and thermal stratification we analyzed in Schneider and Walker (2006).
To elucidate the relative roles extratropical and tropical temperature gradients play in the dynamics of the Hadley circulation, we conducted simulations driven by relaxation of temperatures toward variants of the normative state without a tropical temperature gradient and without an extratropical temperature gradient. The state without a tropical temperature gradient is the same as the normative state except that temperatures are set to the value at $\pm 32^{\circ}$ at latitudes equatorward of $\pm 32^{\circ}$. Conversely, in the state without an extratropical temperature gradient, temperatures are set to the value at $\pm 32^{\circ}$ for latitudes poleward of $\pm 32^{\circ}$. For the modified temperature gradients, the parameter $\Delta_{h}$ refers to the equator-to-pole surface temperature difference that the radiative-equilibrium state would have without modification.

Extending a study of the response of the Hadley circulation to heating displaced off the equator (Walker and Schneider 2005), we also conducted simulations with a variant of the normative radiative-equilibrium state with the maximum surface temperature displaced to the latitude $\phi_{0} \geq 0$ off the equator in the Northern Hemisphere. The surface temperature in radiative equilibrium in these simulations is prescribed as

$$
T_{s}^{e}=260 \mathrm{~K}+\Delta_{h}\left[\cos ^{2} \phi+2 \sin \phi_{0}(1+\sin \phi)\right],
$$

which gives a fixed temperature of $260 \mathrm{~K}$ at the south pole and a maximum temperature of $260 \mathrm{~K}+\Delta_{h}(1+$ $\left.\sin ^{2} \phi_{0}+2 \sin \phi_{0}\right)$ at latitude $\phi_{0}$. We fixed the temperature contrast $\Delta_{h}=120 \mathrm{~K}$ in these simulations. For large $\phi_{0}$, this gives large maximum radiative-equilibrium temperatures, for example $634 \mathrm{~K}$ for $\phi_{0}=50^{\circ}$. In the simulations with varying $\phi_{0}$, to facilitate comparisons with nearly inviscid axisymmetric theories, the thermal relaxation time is constant and equal to 50 days throughout the atmosphere. The radiative-equilibrium temperature contrast of $\Delta_{h}=120 \mathrm{~K}$ with constant thermal relaxation time results, for $\phi_{0}=0$, in an equator-to-pole surface temperature contrast of $32 \mathrm{~K}$ in dynamical equilibrium, comparable with annual-mean or equinox surface temperature contrasts in Earth's atmosphere.

The horizontal resolution of the model was chosen depending on planet radius and rotation rate. A resolution of T42 was used in simulations with a planet radius equal to Earth's radius and in simulations in which the rotation rate is that of Earth or less; T85 was used in simulations with twice Earth's radius or with a rotation rate between one and two times Earth's rotation rate; T127 was used in simulations with four times Earth's rotation rate or radius. In all cases, there were 30 unequally spaced $\sigma$ levels in the vertical. Each simulation was started from a state nearby in parameter 


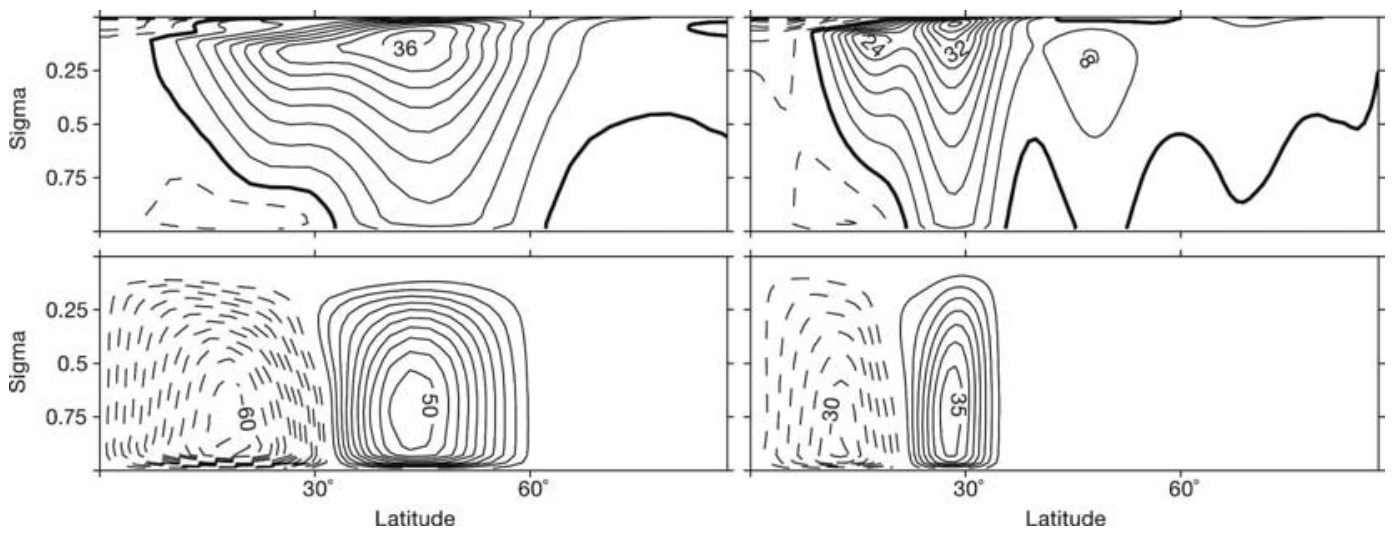

Fig. 3. (top row) Zonal wind and (bottom row) mass flux streamfunction, for case (left column) $\Delta_{h}=90 \mathrm{~K}$, $\gamma=0.7, \Omega=\Omega_{e}$ and (right column) $\Delta_{h}=120 \mathrm{~K}, \gamma=0.7, \Omega=4 \Omega_{e}$ with spatially varying thermal relaxation time. Contour intervals are $4 \mathrm{~m} \mathrm{~s}^{-1}$ and $5 \times 10^{9} \mathrm{~kg} \mathrm{~s}^{-1}$ for zonal wind and streamfunction. Dashed contours indicate easterly wind and clockwise motion. Thick contours in the top row are zero zonal wind contours.

space, typically the end of an earlier simulation. Simulations with T42 resolution were run for 300 days; results shown from these simulations are averaged over the final 200 days. Simulations with T85 resolution were run for 200 days; results are averaged over the final 100 days. Simulations with T127 were run for 250 days; results are an average of the final 150 days (with a day referring to $86400 \mathrm{~s}$, independent of planetary rotation rate).

All temporal and zonal means shown are averages along the model's $\sigma$ surfaces. For the simulations with hemispherically symmetric radiative-equilibrium temperatures, the Northern and Southern Hemispheres are statistically identical. The results shown for these simulations are averages of the Northern and Southern Hemispheres.

\section{Simulations with hemispherically symmetric thermal driving}

We first discuss series of simulations in which we varied planet radius $a$ and rotation rate $\Omega$, convective lapse rate $\gamma \Gamma_{d}$, and the equator-to-pole surface temperature difference $\Delta_{h}$ in the normative radiativeequilibrium state. With Earth's radius $a_{e}$ and rotation rate $\Omega_{e}$ and with the spatially varying thermal relaxation time, the rescaling parameter $\gamma$ was assigned values of $0.6,0.7,0.8,0.9$, and 1.0. For each value of $\gamma$, we conducted simulations with temperature contrasts $\Delta_{h}$ between 15 and $360 \mathrm{~K}$, corresponding to nondimensionalized temperature contrasts $\Delta_{h} / T_{0}$ between 0.06 and 0.72 . The resulting dynamical equilibria of the simulations exhibit equator-to-pole surface temperature contrasts between 12 and $166 \mathrm{~K}$. We also conducted one series of simulations in which we varied the tempera- ture contrast $\Delta_{h}$ between 30 and $360 \mathrm{~K}$, with Earth's radius $a_{e}$ and rotation rate $\Omega_{e}, \gamma=0.7$, and a constant thermal relaxation time of 50 days throughout the atmosphere.

The wide range of parameters produces both Earthlike circulations with one jet in each hemisphere and circulations with multiple jets in each hemisphere (Fig. 3). In the simulations in Fig. 3, the strengths of the Hadley cells and of the Ferrel cells are similar, unlike in the observations (Fig. 2), in which the equinoctial Hadley cells are stronger than the Ferrel cells. However, the strength of the Hadley cells in the simulations increases with increasing convective lapse rate $\gamma \Gamma_{d}$, and, for convective lapse rates greater than those in Fig. 3 $(\gamma=0.7)$, it can be considerably larger than that of the Ferrel cells.

\section{a. Hadley circulation strength}

In the nearly inviscid limit, the potential temperature flux in axisymmetric Hadley circulations with, in the small-angle approximation, a quadratic dependence of radiative-convective equilibrium potential temperatures on latitude scales as $\Delta_{v}^{\prime} \Psi_{\max }^{\prime} \sim\left(H_{t}^{\prime} \Delta_{h}^{\prime}\right)^{5 / 2}$, where $\Delta_{v}^{\prime}=\Delta_{v} / \theta_{0}$ is a nondimensional gross stability and $\Psi_{\max }^{\prime}$ is the maximum absolute value of the mass flux streamfunction nondimensionalized with the parameters in relation (2b).

The potential temperature flux in our simulations displays a more complex scaling behavior. We chose the height $H_{0}=12 \mathrm{~km}$ and the mean surface temperature $T_{0}=260 \mathrm{~K}+2 / 3 \Delta_{h}$ in radiative equilibrium for the nondimensionalization $H_{t}^{\prime}=H_{t} / H_{0}$ and $\Delta_{h}^{\prime}=\Delta_{h} / T_{0}$. Figure 4 shows, as a function of $H_{t}^{\prime} \Delta_{h}^{\prime}$, the potential temperature flux at the latitude at which the Hadley circulation streamfunction attains its maximum abso- 

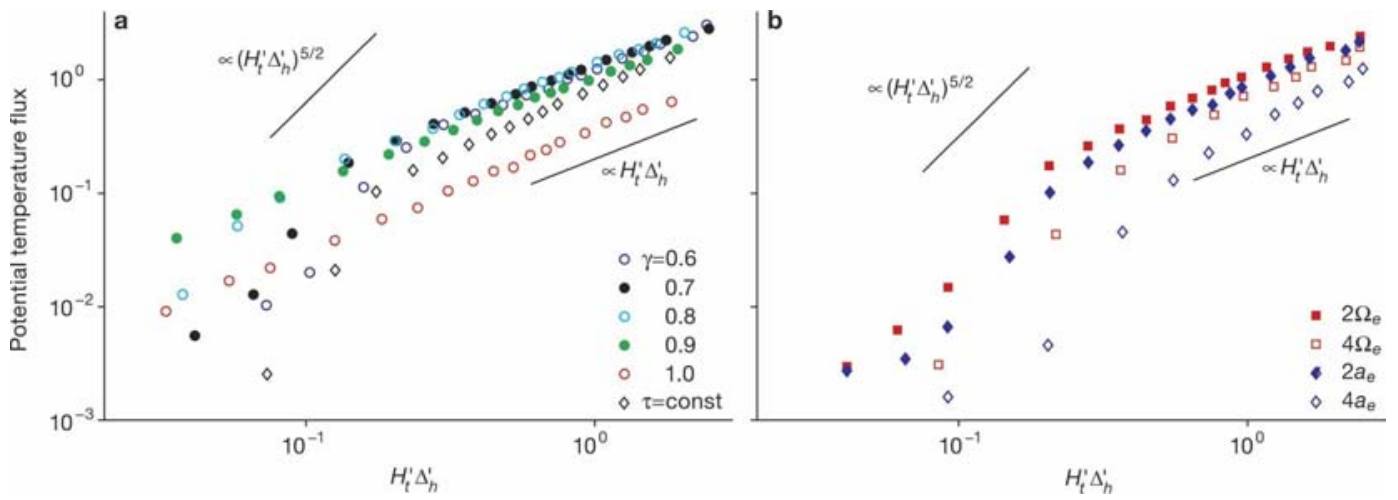

FIG. 4. Nondimensional potential temperature flux at the latitude of maximum absolute value of the Hadley circulation streamfunction, integrated over the depth of the atmosphere and nondimensionalized such that a flux of one nondimensional unit, if multiplied by the specific heat $c_{p}$, corresponds to a poleward energy flux of $4.8 \mathrm{PW}$. Lines show power laws with exponents 1 and 5/2. (a) Simulations with Earth's radius and rotation rate and with spatially varying thermal relaxation time and convective lapse rates $\gamma \Gamma_{d}(\gamma=0.6, \ldots, 1.0)$ and with constant thermal relaxation time $(\tau=$ const $)$ and Earth-like convective lapse rate $(\gamma=0.7)$. (b) Simulations with an Earth-like convective lapse rate $(\gamma=0.7)$, with spatially varying thermal relaxation time, and with radius and rotation rate two and four times the radius and rotation rate of Earth.

lute value. ${ }^{1}$ As seen in the figure, simulations with $\gamma=$ 0.9 and 1.0 show a roughly linear dependence of potential temperature flux on $H_{t}^{\prime} \Delta_{h}^{\prime}$, while the scaling for the $\gamma=0.6,0.7$, and 0.8 simulations in Fig. 4a and for all of the simulations in Fig. $4 \mathrm{~b}$ is in two regimes: a steeper dependence for smaller $H_{t}^{\prime} \Delta_{h}^{\prime}$ and a roughly linear dependence for larger $H_{t}^{\prime} \Delta_{h}^{\prime}$. The scaling behavior of the

\footnotetext{
${ }^{1}$ The potential temperature flux is integrated over the depth of the atmosphere; the maximum absolute value of the Hadley circulation streamfunction refers to the maximum absolute value of the mass flux streamfunction above the level $\sigma=0.7$ (i.e., above the model's planetary boundary layer); and the tropopause height is determined as described by Schneider and Walker (2006). Determining the tropopause height according to the lapse-rate criterion of the World Meteorological Organization, instead of in the way described by Schneider and Walker (2006), or integrating the potential temperature flux from the surface to the tropopause, instead of to the top of the atmosphere, does not substantially change the results. Moreover, for a more direct comparison with $\mathrm{HH}$, the temperature contrast $\Delta_{h}^{\prime}$ should be based on radiativeconvective equilibrium potential temperatures vertically averaged over the troposphere. This is not identical with the nondimensional temperature contrast we use, which is based on radiativeequilibrium surface temperatures. However, by computing radiative-convective equilibrium states of the simulations (cf. Schneider and Walker 2006) and estimating potential temperature contrasts and mean potential temperatures from them, we have verified that the nondimensional temperature contrast based on vertically averaged potential temperatures in radiative-convective equilibrium scales roughly linearly with the nondimensional temperature contrast based on surface temperatures in radiative equilibrium, so that the scaling behavior of the simulations does not change substantially if expressed in terms of vertically averaged potential temperatures in radiative-convective equilibrium.
}

simulations with constant thermal relaxation time and $\gamma=0.7$ is similar to that of the simulations with spatially varying thermal relaxation time and $\gamma=0.7$, demonstrating that spatial variations of the thermal relaxation time are not primarily responsible for the discrepancies between $\mathrm{HH}$ scaling and that exhibited by our simulations. The differences between the radiative-convective equilibrium state of our simulations and that of the $\mathrm{HH}$ model, for example, in the vertical thermal structure, also do not affect the expected scaling behavior in the nearly inviscid axisymmetric limit substantially, and the Hadley circulation extent in all simulations is small enough that the small-angle approximation is likewise not responsible for the discrepancies (cf. Fig. 7 below). ${ }^{2}$

\footnotetext{
${ }^{2}$ By numerical calculations analogous to those of $\mathrm{HH}$ but with the radiative-convective equilibrium state of our simulations, we have verified that HH's nearly inviscid axisymmetric theory for our simulations with constant thermal relaxation time leads to the scaling relations (2) for small Hadley circulation extent. (The convection scheme, whether interacting with the circulation or modifying the radiative-convective equilibrium state, does not substantially affect the scaling relations. It merely redistributes enthalpy vertically, and the scaling relations depend only on vertically averaged radiative-convective equilibrium potential temperatures.) In the nearly inviscid axisymmetric limit, the scaling of the extent of the Hadley circulations is consistent with the scaling relation (2a) over the entire range of parameters of our simulations. The scaling of the strength is close to the scaling relation (2b) for small $H_{t}^{\prime} \Delta_{h}^{\prime}$ and somewhat closer to the quadratic scaling $\Psi_{\max } \sim$ $\left(H_{t}^{\prime} \Delta_{h}^{\prime}\right)^{2}$ for larger $H_{t}^{\prime} \Delta_{h}^{\prime}$. The regime transition and the roughly linear strength scaling seen in the simulations for larger $H_{t}^{\prime} \Delta_{h}^{\prime}$ cannot be accounted for by HH's nearly inviscid axisymmetric theory.
} 
The discrepancies between HH scaling and that exhibited by our simulations can be understood by examining the local Rossby number Ro $=-\bar{\zeta} / f$ (Fig. 5), which, in the horizontal upper branches of the circulations, gives a measure of the relative importance of nonlinear momentum advection by the mean circulation, as seen from the zonal momentum balance (1). (The overbar now denotes a temporal and zonal mean along $\sigma$ surfaces.) In regions where the local Rossby number is small, the mean circulation responds linearly to changes in eddy forcing. This is the case in the extratropical upper tropospheres of all three circulations shown in Fig. 5, where eddy momentum flux convergence is balanced by the meridional advection of planetary angular momentum by (or the Coriolis force on) the Ferrel circulations. The local Rossby number is also relatively small $(0.2 \lesssim$ Ro $\lesssim 0.4)$ in the upper branches of the Hadley circulations in most simulations, as demonstrated by Figs. 5b,c, indicating that eddy momentum flux divergence is to a large extent balanced by the meridional advection of planetary angular momentum by the Hadley circulation.

Figure 5a shows the local Rossby number for a simulation in which a relatively small radiative-equilibrium temperature contrast of $\Delta_{h}=15 \mathrm{~K}$ produces only weak eddies and, consequently, a poleward flow in the upper branch of the Hadley circulation that is closer to angular momentum-conserving. The local Rossby number is greater than 0.4 throughout the upper branch of the Hadley circulation and greater than 0.8 in the deep Tropics, suggesting that nearly inviscid axisymmetric theories might be more relevant in this case. However, the flow in the Hadley circulation is more complex than that described by $\mathrm{HH}$, as a small counterrotating circulation forms near the top of the Hadley circulation in the deep Tropics.

The balance between advection of planetary angular momentum by the Hadley circulation and eddy momentum flux divergence is made explicit in Fig. 6, which shows that, for many simulations, the strength of the Hadley circulation $\Psi_{\max }$ scales with the quantity

$$
\mathcal{T}=\frac{2 \pi a}{f g} \int_{\sigma_{\max }}^{0} \boldsymbol{\nabla} \cdot\left[\bar{p}_{s} \cos \phi\left(\overline{{\overline{u^{\prime} v^{\prime}}}^{\sigma}}, \overline{\bar{u}^{\prime} \omega^{\prime}}\right)\right] d \sigma,
$$

where $\omega=D \sigma / D t$ is the vertical velocity in $\sigma$ coordinates. [The symbol $\overline{(\cdot)}^{\sigma}=\overline{\left(p_{s} \cdot\right)} / \bar{p}_{s}$ denotes a surfacepressure weighted temporal and zonal mean along $\sigma$ surfaces, and primes denote deviations therefrom.] The lower boundary $\sigma_{\max }$ of the integration is the level at which the Hadley circulation streamfunction attains its maximum absolute value, and $\mathcal{T}$ is evaluated at the latitude of this maximum. The quantity $f \mathcal{T} /(2 \pi a \cos \phi)$

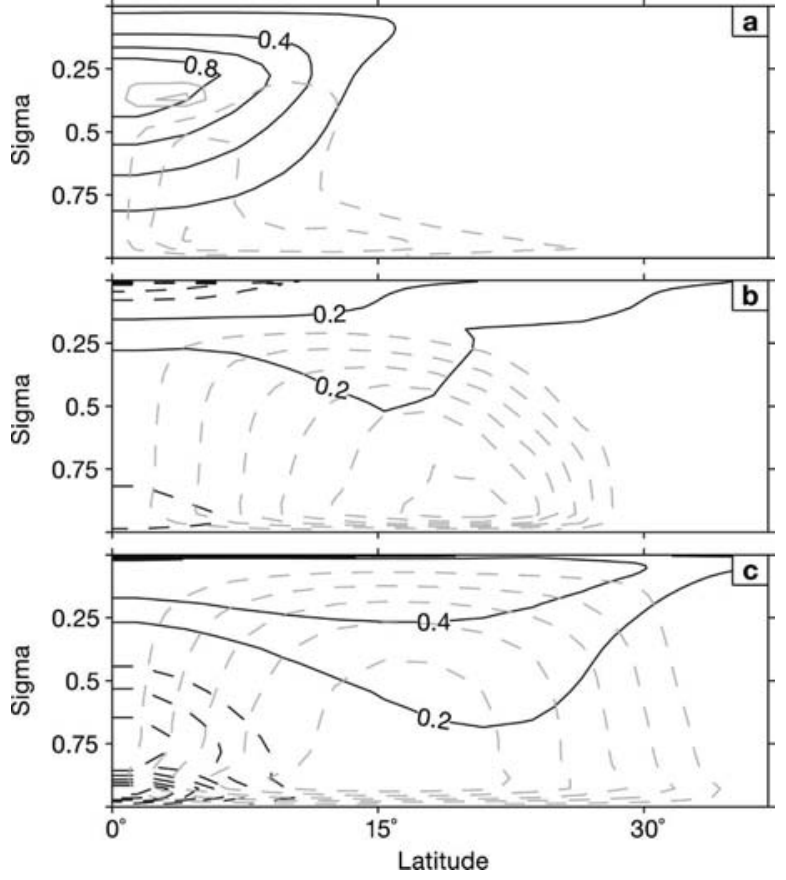

FIG. 5. Local Rossby number $($ Ro $=-\bar{\zeta} / f)$ for simulations with $\gamma=0.7$ and (a) $\Delta_{h}=15 \mathrm{~K}$, (b) $\Delta_{h}=45 \mathrm{~K}$, and (c) $\Delta_{h}=360 \mathrm{~K}$ with spatially varying thermal relaxation time. Gray contours show mass flux streamfunction with contour intervals of $0.5,10$, and $25 \times 10^{9} \mathrm{~kg} \mathrm{~s}^{-1}$ for (a), (b), and (c), respectively.

is a mass-weighted vertical integral of the eddy momentum flux divergence that appears in the $\sigma$-coordinate counterpart of the zonal momentum balance (1). When Ro $\rightarrow 0$, the Hadley circulation strength $\Psi_{\max }$ is approximately equal to $\mathcal{T}$ at the latitude of the streamfunction extremum, as can be seen by integrating the $\sigma$-coordinate counterpart of the zonal momentum balance (1) from the top of the atmosphere downward to the level $\sigma_{\max }$ and multiplying by the circumference $2 \pi a \cos \phi$ of latitude circles. ${ }^{3}$ In many simulations, spanning nearly four orders of magnitude of Hadley circulation strength and including different convective lapse rates, planet radii, and rotation rates, the Hadley circulation strength $\Psi_{\max }$ is indeed approximately equal to $\mathcal{T}$ or at least scales with $\mathcal{T}$. Consistent with its large Rossby number in the upper branch of the Hadley circulation, the simulation depicted in Fig. 5a does not fall

\footnotetext{
${ }^{3}$ Even if Ro $\rightarrow 0$ and if the mean vertical mass flux vanishes throughout the atmospheric column at the Hadley circulation extremum, so that the mean vertical advection of angular momentum vanishes, the equality between $\Psi_{\max }$ and $\mathcal{T}$ is only approximate. The form drag at the level $\sigma_{\max }$, owing to correlations between the surface pressure and the pressure gradient force in $\sigma$ coordinates, also contributes to the zonal momentum balance, but it is usually small.
} 

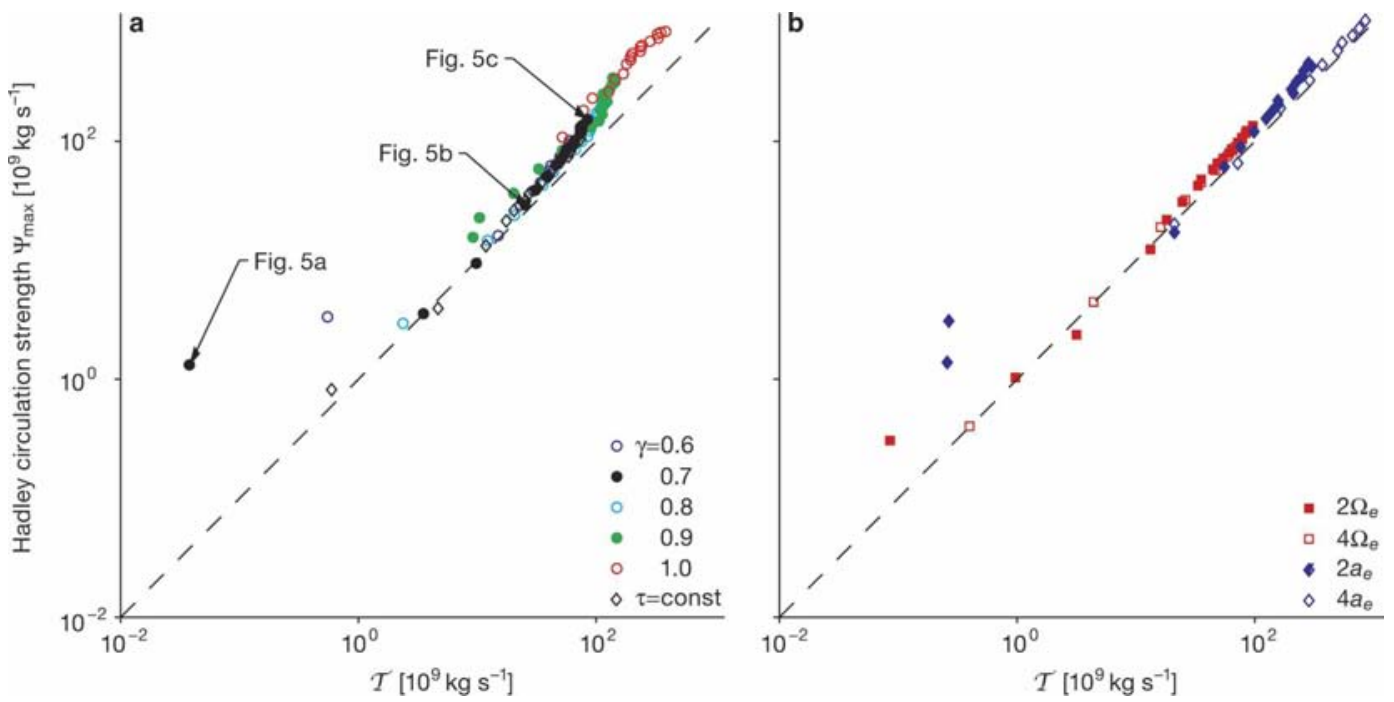

FIG. 6. Hadley circulation strength $\Psi_{\max }$ as a function of the quantity $\mathcal{T}$, which is proportional to an integrated eddy momentum flux divergence (4) at the latitude of maximum absolute value of the Hadley circulation streamfunction. The dashed line shows the identity $\Psi_{\max }=\mathcal{T}$. Simulations in (a) and (b) are the same as in Figs. 4a,b.

on the line $\Psi_{\max }=\mathcal{T}$. The other simulations that deviate from the line $\Psi_{\max }=\mathcal{T}$ also show relatively large local Rossby numbers in the upper branches of the Hadley circulations. For example, the Hadley circulations in the simulations with a dry-adiabatic convective lapse rate $(\gamma=1.0)$ have local Rossby numbers of about 0.5 in their upper branches in the vicinity of the latitude of the streamfunction extrema. In these simulations, the thermal stratification within the Hadley circulations is nearly statically neutral, and the Hadley circulations are up to an order of magnitude stronger than Earth's annual-mean or equinox Hadley circulation.

The contribution of the vertical eddy momentum flux ${\overline{u^{\prime} \omega^{\prime}}}^{\sigma}$ to $\mathcal{T}$ is negligible in most simulations. An exception are the simulations with $\gamma=1.0$, in which the vertical momentum flux contribution, probably at least in part owing to convection on the model's grid scale, dominates $\mathcal{T}$. (Poleward of the latitudes of the Hadley circulation extrema at which $\mathcal{T}$ is evaluated, the horizontal eddy momentum flux divergence again dominates the vertical eddy momentum flux divergence in the interior atmosphere in these simulations.)

The approximately linear relation between Hadley circulation strength and eddy momentum flux divergence helps to explain the regime transition in scaling behavior seen in the potential temperature flux (Fig. 4). Since over a wide range of flow parameters, the Hadley circulation strength $\Psi_{\max }$ is approximately linearly related to the eddy momentum flux divergence, the potential temperature flux $\Delta_{v} \Psi_{\max }$ is likewise directly related to the eddy momentum flux divergence, with the gross stability $\Delta_{v}$ primarily controlled by the convective lapse rate and, through its influence on the tropopause height, the tropical surface temperature. The gross stability exhibits no transition in scaling behavior as a function of $H_{t}^{\prime} \Delta_{h}^{\prime}$, so the transition between the two scaling regimes of the potential temperature flux is a transition in scaling regimes of the eddy momentum flux divergence. The transition between the two regimes occurs where the supercriticality of the extratropical thermal stratification and of baroclinic eddies reaches saturation (Schneider and Walker 2006). In the low $H_{t}^{\prime} \Delta_{h}^{\prime}$ regime, the extratropical thermal stratification is primarily maintained by convection. In the high $H_{t}^{\prime} \Delta_{h}^{\prime}$ regime, the extratropical thermal stratification is modified by baroclinic eddies. As $\gamma$ is increased, the transition between the regimes in which convection and eddies dominate the maintenance of the extratropical thermal stratification occurs at smaller temperature contrasts $\Delta_{h}$, and thus at smaller $H_{t}^{\prime} \Delta_{h}^{\prime}$, such that the $\gamma$ $=0.9$ and 1.0 simulations are all in the eddy-dominated regime (Schneider and Walker 2006). Scaling laws of baroclinic eddy fluxes change at this regime transition, and they are imprinted on the scaling behavior of the Hadley circulation. (We will discuss scaling laws of baroclinic eddy fluxes elsewhere.)

The approximately linear relation between Hadley circulation strength and eddy momentum flux divergence implies that it is a coincidence that the scaling of the potential temperature flux in the low $H_{t}^{\prime} \Delta_{h}^{\prime}$ regime in some series of simulations in Fig. 4 is consistent with the HH scaling $\Delta_{v}^{\prime} \Psi_{\max }^{\prime} \sim\left(H_{t}^{\prime} \Delta_{h}^{\prime}\right)^{5 / 2}$. This coincidence is 


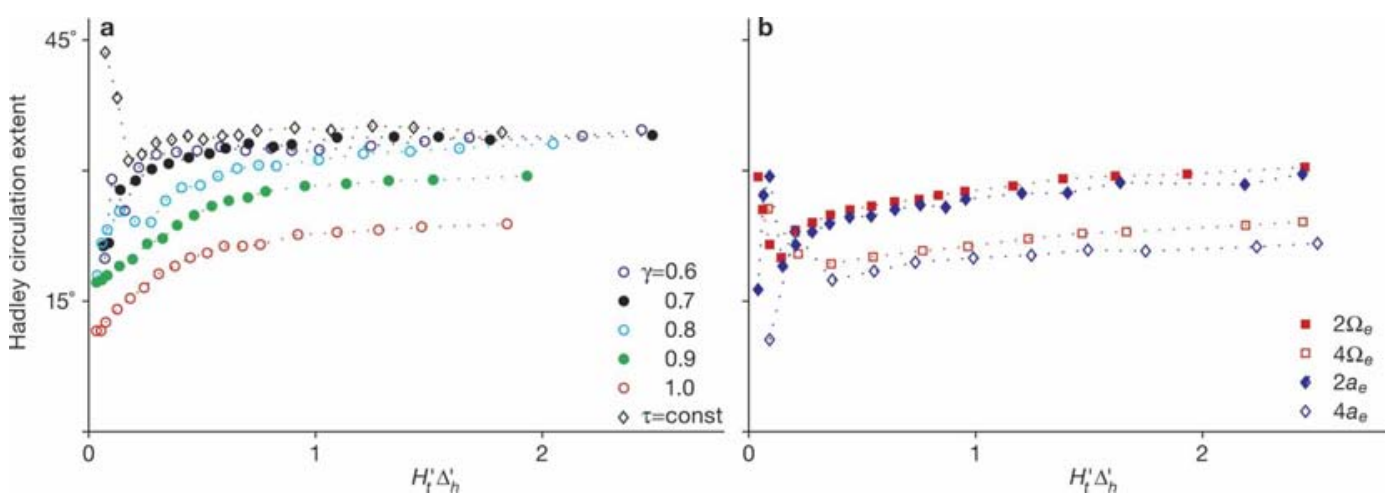

FIG. 7. Hadley circulation extent $\phi_{H}$ as a function of $H_{t}^{\prime} \Delta_{h}^{\prime}$. Simulations in (a) and (b) are the same as in Figs. 4a,b.

brought about by a similar scaling of the eddy momentum flux divergence. Other than for the smallest temperature gradients, the simulations in question all exhibit relatively small local Rossby numbers in the upper branches of the Hadley circulations (see, e.g., Fig. 5b).

Figure 4 a shows that in the eddy-dominated regime, in the simulations with spatially varying thermal relaxation time, the potential temperature flux depends little on the convective lapse rate $\gamma \Gamma_{d}$ for $\gamma<1.0$. This invariance of the potential temperature flux stems from the fact that, in the eddy-dominated regime, there appears to be a cancellation between changes in gross stability and changes in Hadley circulation strength with convective lapse rate. In light of Fig. 6a, the mechanism behind this cancellation must involve changes in $\mathcal{T}$ with convective lapse rate. These changes in $\mathcal{T}$ appear to be, at least in part, due to changes in Hadley circulation extent with convective lapse rate (see below), which affect $\mathcal{T}$ through the Coriolis parameter evaluated at the latitude of the streamfunction extremum [cf. Eq. (4)]. However, we do not fully understand the mechanisms behind the near independence of potential temperature flux from convective lapse rate in this set of simulations; it may be a coincidence.

The potential temperature fluxes in the $\gamma=1.0$ simulations are all in the eddy-dominated regime and scale roughly linearly with $H_{t}^{\prime} \Delta_{h}^{\prime}$. However, the flux is weaker than in simulations with $\gamma<1.0$ (Fig. 4a). When $\gamma=$ 1.0 , the tropical lapse rate is approximately dry adiabatic, so the gross stability $\Delta_{v}$ approaches zero and leads to relatively small potential temperature fluxes, despite some compensating increase in Hadley circulation strength for $\gamma=1.0$ compared with $\gamma<1.0$ (Fig. 6a).

The results in Fig. 6 show that the scaling behavior of the potential temperature flux and Hadley circulation strength in the simulations cannot be expected to be captured by nearly inviscid axisymmetric theories. The approximately linear relation between Hadley circula- tion strength and eddy momentum flux divergence over a wide range of flow parameters is more consistent with linear theories of the Hadley circulations for the limit $\mathrm{Ro} \rightarrow 0$. However, in some simulations, such as those with convective lapse rates $\gamma \geq 0.9$ and strong Hadley circulations or with weak baroclinic eddies (Fig. 6), nonlinear momentum advection by the mean circulation also appears to be important.

\section{b. Hadley circulation extent}

In the nearly inviscid limit, the extent of axisymmetric Hadley circulations with, in the small-angle approximation, a quadratic dependence of radiative-convective equilibrium potential temperatures on latitude, according to relation $(2 \mathrm{a})$, scales as $\phi_{H} \sim\left(H_{t}^{\prime} \Delta_{h}^{\prime}\right)^{1 / 2}$. As seen in Fig. 7, our simulations do not exhibit a simple power-law relation between Hadley circulation extent ${ }^{4}$ and $H_{t}^{\prime} \Delta_{h}^{\prime}$. Instead, with the exception of simulations at low $H_{t}^{\prime} \Delta_{h}^{\prime}$ with constant thermal relaxation time or with two and four times Earth's radius or rotation rate, the Hadley circulation extent increases rapidly for $H_{t}^{\prime} \Delta_{h}^{\prime} \lesssim$ 0.5 and more slowly thereafter-roughly as $\left(H_{t}^{\prime} \Delta_{h}^{\prime}\right)^{1 / 8}$ for the narrower Hadley circulations for $H_{t}^{\prime} \Delta_{h}^{\prime} \gtrsim 0.5$ and more slowly for the wider ones. The extent also displays a dependence on convective lapse rate: smaller values of $\gamma$, which are associated with statically more

\footnotetext{
${ }^{4}$ The Hadley circulation extent is determined as the first latitude poleward of the maximum absolute value of the Hadley circulation streamfunction at which the mass flux streamfunction at the $\sigma$ level of its extremum above $\sigma=0.7$ is $10 \%$ of its extremal value. This latitude is typically close to the latitude at which the streamfunction changes sign. In axisymmetric or nearly axisymmetric simulations, however, the latitude at which the streamfunction is $10 \%$ of is extremal value gives a more reliable diagnostic of Hadley circulation extent because in those simulations the streamfunction may not change sign near the poleward boundary of the Hadley circulation, although this poleward boundary is relatively well-defined.
} 
stable convective lapse rates, yield wider Hadley circulations. In contrast, the Hadley circulation extent in the $\mathrm{HH}$ model does not depend explicitly on convective lapse rate or gross stability $\Delta_{v}^{\prime}$. These results show that it must be regarded a coincidence that, for a radiativeequilibrium temperature contrast that results in Earthlike atmospheric circulations, the $\mathrm{HH}$ model yields a Hadley circulation extent similar to that in Earth's atmosphere (according to HH's exact expressions, $\phi_{\mathrm{H}}=32^{\circ}$ for $\Delta_{h}=100 \mathrm{~K}, \theta_{0}=295 \mathrm{~K}$, and $H_{t}=15 \mathrm{~km}$, independent of tropical lapse rates).

Aspects of the scaling behavior seen in Fig. 7 are qualitatively consistent with the idea, discussed, for example, by Held (2000), that baroclinic instability may limit the meridional extent of the Hadley circulation. This idea can be explored using the necessary condition for instability in the quasigeostrophic two-layer model, in which the flow can be baroclinically unstable when the vertically averaged temperature gradient exceeds the critical value $\partial_{\phi} T_{c}=-a(\beta / f) \Delta_{v}$. [Since the existence of this critical temperature gradient is an artifact of the vertical truncation of the two-layer model, such considerations are necessarily heuristic; however, they can be made more precise using the supercriticality concept of Schneider and Walker (2006).] The lowest latitude at which the geopotential gradient balancing the angular momentum-conserving zonal wind exceeds the baroclinic geopotential gradient corresponding to the critical temperature gradient may represent an upper bound on the extent of the Hadley circulation. (Since the baroclinic component of the geopotential is a vertical average of temperature, a critical vertically averaged temperature gradient implies a critical baroclinic geopotential gradient.) This latitude, in the small-angle approximation, scales as

$$
\phi_{c} \sim\left(R \Delta_{v}^{\prime}\right)^{1 / 4}
$$

see Held (2000) and Schneider (2006). Qualitatively consistent with this heuristic critical latitude for baroclinic instability, the Hadley circulation extent for larger values of $H_{t}^{\prime} \Delta_{h}^{\prime}$ shown in Fig. 7 is nearly independent of $\Delta_{h}^{\prime}$ and increases with gross stability $\Delta_{v}^{\prime}$, albeit more slowly than with $\left(\Delta_{v}^{\prime}\right)^{1 / 4}$ : Since gross stability changes in the simulations are dominated by lapse rate changes, the gross stability scales roughly as $(1-\gamma)$ at fixed $\Delta_{h}$, so according to relation (5), one would expect the Hadley circulation extent to increase by a factor of about $\sqrt{2}$ as $\gamma$ is reduced from 0.9 to 0.6 , but the actual increase is by less than a factor of 1.2. (Taking changes in tropopause height into account, which enter the factor $R=g H_{t} /\left(\Omega^{2} a^{2}\right)$, does not improve the quantitative agreement with relation (5) because the simulations with smaller convective lapse rates and greater gross stabilities also have higher tropopauses.) It may also be tempting to conclude that at smaller values of $H_{t}^{\prime} \Delta_{h}^{\prime}$ the Hadley circulation extent is that given by HH's nearly inviscid axisymmetric theory, so that the circulation extent is in two regimes: one at smaller $H_{t}^{\prime} \Delta_{h}^{\prime}$ in which the Hadley circulation extent is set by the nearly inviscid axisymmetric theory, and one at higher $H_{t}^{\prime} \Delta_{h}^{\prime}$ in which the extent is limited by baroclinic instability (cf. Held 2000). As shown in Figs. 5 and 6, however, even at relatively small meridional temperature gradients, the Hadley circulations are not close to angular momentum-conserving. Furthermore, neither theory correctly captures the dependence of the extent on the rotation rate discussed in section $4 \mathrm{c}$.

Simulations with a jump in Hadley circulation extent at low $H_{t}^{\prime} \Delta_{h}^{\prime}$ highlight a transition between regimes with insignificant and significant eddy dynamics. For example, Figs. 8a-f show this transition for simulations with four times Earth's radius. When relaxed toward a radiative-equilibrium state with $\Delta_{h}=30 \mathrm{~K}$ (Figs. 8a,b), the resulting dynamical-equilibrium temperature gradient is not sufficient to generate significant eddies, giving a narrow and weak Hadley circulation and a weak jet with center near $10^{\circ}$.

The next-higher value $\Delta_{h}=60 \mathrm{~K}$ produces a dynamical equilibrium that is baroclinically unstable and leads to the generation of a second jet centered near $27^{\circ}$ and weak additional jets at higher latitudes (Figs. 8c,d). Latitudes equatorward of about $12^{\circ}$ remain relatively free of eddies. The combination of weak eddies poleward of about $12^{\circ}$ and relatively eddy-free lower latitudes leads to a thermally direct circulation extending to roughly $22^{\circ}$ : a circulation within $12^{\circ}$ of the equator that is closer to angular momentum-conserving, merged with an eddy-driven circulation that extends to the equatorward flank of the second jet.

At larger values of $\Delta_{h}$, the jet centered near $10^{\circ}$ and the second jet merge and strengthen. The extratropical jets become associated with stronger eddy momentum flux convergence and surface westerlies (Figs. 8e,f). Eddies propagate farther equatorward of the combined jet, to their critical lines on the equatorward flank of the jet, leading to an equatorward shift of the region of eddy momentum flux divergence. The combined jet moves equatorward of the position of the original second, baroclinically unstable jet, as does the region of eddy momentum flux convergence and the associated Ferrel cell, reducing the extent of the Hadley circulation. Similar progressions of Hadley circulation extent are manifested in all simulations in which the eddy scale 


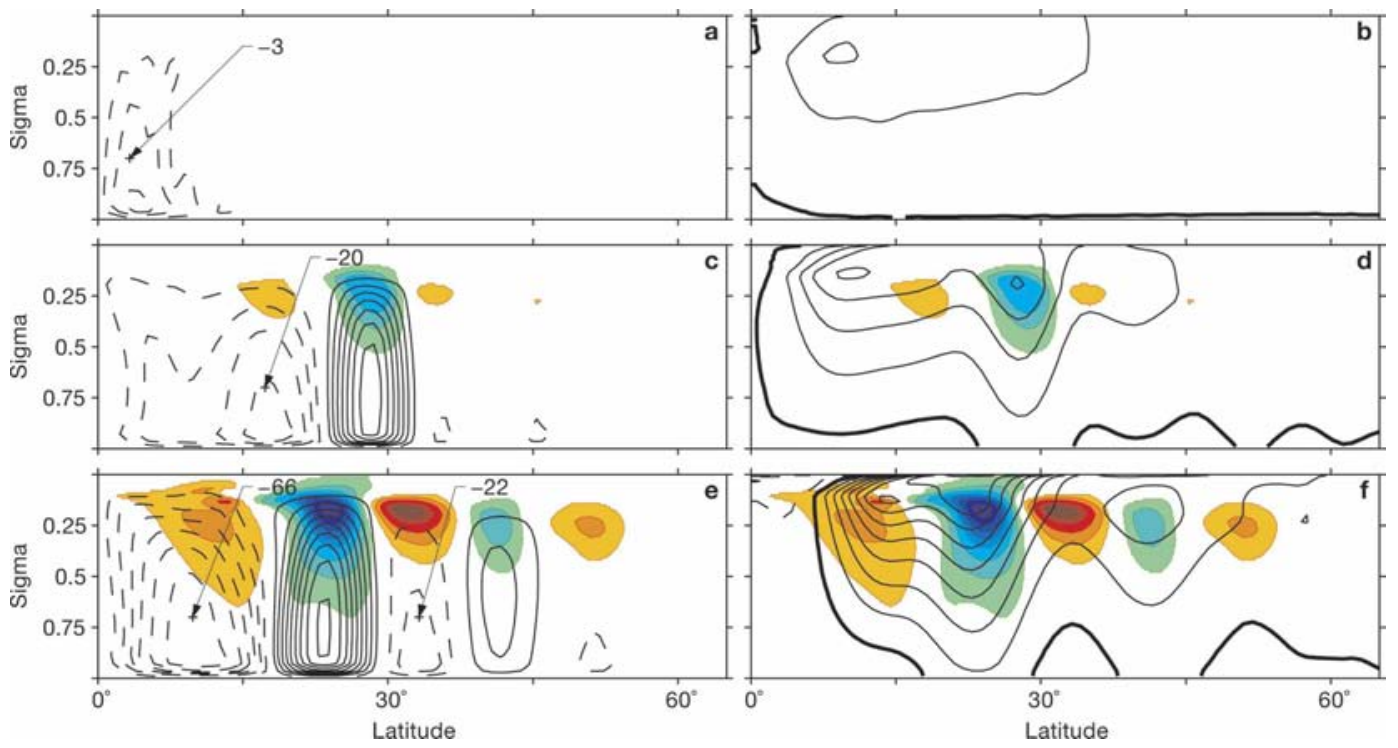

FIG. 8. Simulations with (top row) $\Delta_{h}=30 \mathrm{~K}$, (middle row) $60 \mathrm{~K}$, and (bottom row) $90 \mathrm{~K}$. (left column) Mass flux streamfunction and (right column) zonal wind (zero contour thick). Colored contours show eddy momentum flux divergence [integrand in Eq. (4) divided by $\bar{p}_{s}$ ], with orange/red tones indicating positive values and green/blue tones indicating negative values. In all simulations, $a=4 a_{e}$ and $\gamma=0.7$. Contour intervals for streamfunction are (a) $10^{9} \mathrm{~kg} \mathrm{~s}^{-1}$, (c) $5 \times 10^{9} \mathrm{~kg} \mathrm{~s}^{-1}$, and (e) $10 \times 10^{9} \mathrm{~kg} \mathrm{~s}^{-1}$. In all panels, the contour intervals for zonal wind and eddy momentum flux divergence are $4 \mathrm{~m} \mathrm{~s}^{-1}$ and $1.25 \times 10^{-6} \mathrm{~m} \mathrm{~s}^{-2}$. No eddy momentum flux divergence is visible at this contour interval for the $\Delta_{h}=30 \mathrm{~K}$ simulation; the largest value for this case is approximately $3 \times 10^{-9} \mathrm{~m} \mathrm{~s}^{-2}$. Local extrema of thermally direct streamfunction cells above $\sigma=0.7$ are indicated in units of $10^{9} \mathrm{~kg} \mathrm{~s}^{-1}$.

is sufficiently small, relative to the size to the domain, that there is room for multiple jets.

\section{c. Rotation rate variation}

We investigated how the Hadley circulation strength and extent depend on the planetary rotation rate by fixing $\gamma=0.7$ and $\Delta_{h}=120 \mathrm{~K}$, using the spatially varying thermal relaxation time, and conducting a series of simulations with rotation rates of $4.0,2.0, \sqrt{2}, 1 / \sqrt{2}$, $1 / 2,1 / 4,1 / 8,1 / 16,1 / 32$, and 1/64 times Earth's rotation rate. Consistent with previous studies (e.g., Williams 1988a,b; Navarra and Boccaletti 2002), the Hadley circulation becomes wider and stronger as the rotation rate decreases (Fig. 9). These dependences are made explicit in Figs. 10 and 11, which reveal that the strength scales roughly as $\Omega^{-1 / 3}$ for smaller and as $\Omega^{-2 / 3}$ for larger rotation rates and that the extent scales roughly as $\Omega^{-1 / 5}$ for smaller and as $\Omega^{-1 / 3}$ for larger rotation rates. (We give these power laws as references for comparisons, not to imply that the dependences of Hadley circulation strength and extent on rotation rate necessarily are power laws, rather than, e.g., transcendental functions.) The dependences of strength and extent on rotation rate are considerably weaker than those given by the nearly inviscid axisymmetric scaling relations (2), which are valid where the small-angle approxima- tion is adequate $\left(\Omega \gtrsim \Omega_{e}\right)$ and according to which strength and extent scale as $\Omega^{-3}$ and $\Omega^{-1}$, respectively. The dependence of extent on rotation rate is also weaker than that obtained by the heuristic arguments assuming that baroclinic instability limits the extent of an angular momentum-conserving upper branch of the Hadley circulation, which predict, as long as the smallangle approximation is adequate, that the extent scales as $\Omega^{-1 / 2}$ [cf. Eq. (5)].

For larger rotation rates, the reason for the deviation of the rotation rate scalings from those predicted by nearly inviscid axisymmetric theories is again that the upper branches of the simulated Hadley circulations have local Rossby numbers significantly less than one. The local Rossby numbers in the upper branches of the Hadley circulations generally increase as the rotation rate decreases, as can be inferred from the angular momentum contours in Fig. 9. The upper branches of the circulations for rotation rates $\Omega=\Omega_{e} / 32$ and smaller are nearly angular momentum-conserving around the latitude of the Hadley streamfunction extremum (cf. Fig. 9). The Hadley circulations in these simulations are so wide that the small-angle approximation is no longer adequate. For such wide circulations, the dependence of Hadley circulation strength and extent on rotation rate according to HH's nearly inviscid axisymmetric 

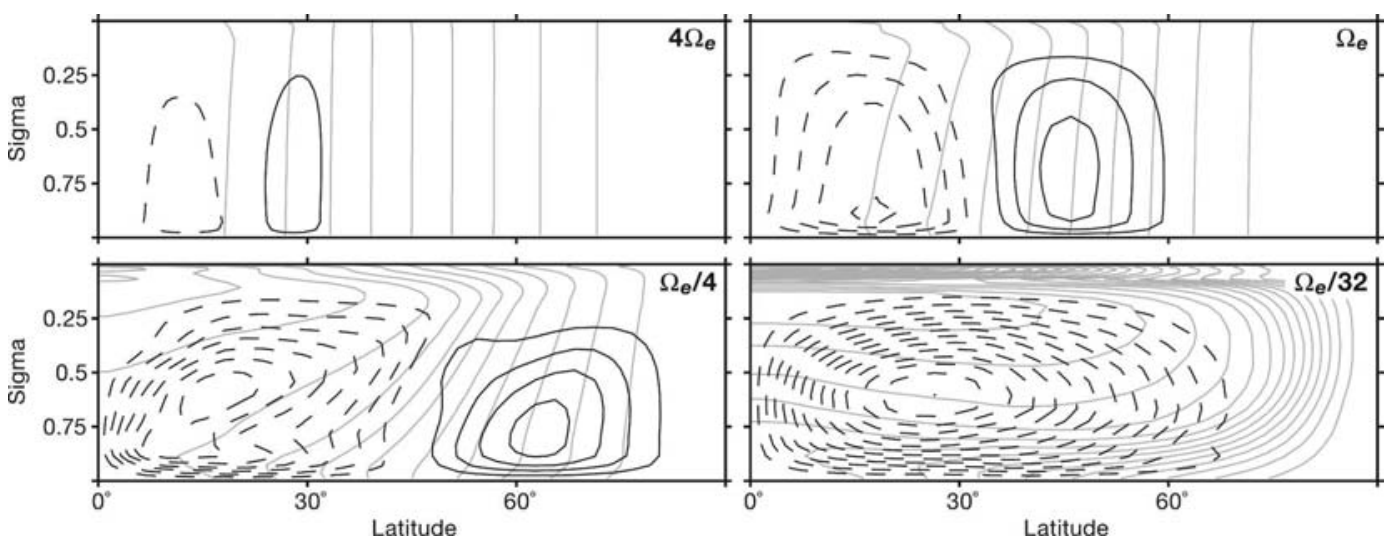

Fig. 9. Mass flux streamfunction (black contours) and angular momentum (gray contours) for simulations with rotation rates $4,1,1 / 4$, and $1 / 32$ times Earth's rotation rate $\Omega_{e^{*}}$. Contour intervals are $20 \times 10^{9} \mathrm{~kg} \mathrm{~s}^{-1}$ and $0.1 \Omega a^{2}$ for streamfunction and angular momentum. In all cases, $\Delta_{h}=120 \mathrm{~K}$ and $\gamma=0.7$.

theory no longer follows power laws. By numerical calculations analogous to those of $\mathrm{HH}$, with the radiativeconvective equilibrium state of our simulations but using a constant thermal relaxation time, we found that Hadley circulations in the nearly inviscid limit extend to latitudes greater than $75^{\circ}$ for rotation rates less than about $\Omega_{e} / 8$ and essentially extend to the poles for rotation rates less than about $\Omega_{e} / 32$. Their strength increases more slowly than $\Omega^{-1 / 3}$ for rotation rates less than about $\Omega_{e} / 8$. (These scaling results for the nearly inviscid axisymmetric limit are unchanged if the radiative-convective equilibrium state of the $\mathrm{HH}$ model is used.) Thus, the nearly inviscid axisymmetric theory is still not quantitatively consistent with the results in Figs. 10 and 11, probably because eddy momentum fluxes in the simulated circulations lead to deviations from angular momentum conservation in the ascending and descending branches, which are broad, rather than being confined to narrow latitude belts as assumed in HH's nearly inviscid axisymmetric theory (cf. Fig. 9). The spatial variations of the thermal relaxation time in our simulations may also contribute to the quantitative differences to HH's nearly inviscid axisymmetric theory.

The simulations with low rotation rates $\left(\Omega \leq \Omega_{e} / 4\right)$ exhibit equatorial superrotation, that is, local angular momentum maxima in the equatorial tropospheresimilar to what is seen, for example, in Venus' atmosphere. The local angular momentum maximum can be seen, for example, in the angular momentum contours for the simulation with $\Omega=\Omega_{e} / 32$ in Fig. 9. Equatorial superrotation is also evidence for the significance of eddy momentum fluxes in the circulations. The local angular momentum maximum in the interior atmosphere is maintained by eddy momentum fluxes into the maximum; it could not be generated by axisymmet- ric circulations (cf. Hide 1969; Schneider 1977; Held and Hou 1980).

\section{d. Tropical versus extratropical temperature gradients}

To elucidate the relative roles tropical and extratropical temperature gradients play in the dynamics of the Hadley circulation, we conducted series of simulations relaxed toward two additional radiative-equilibrium states, one with flat tropical temperatures and one with flat extratropical temperatures. In these series of simulations, we set $\gamma=0.7$, used the spatially varying thermal relaxation time, and varied the temperature contrast $\Delta_{h}$ in the same way as for the series of simulations with the normative radiative-equilibrium states. As described in section 3 , in the radiative-equilibrium states with flat tropical (or extratropical) temperatures, temperatures are constant equatorward (or poleward) of $\pm 32^{\circ}$, where $32^{\circ}$ is the latitude up to which the Hadley circulation extends in the simulation with the normative radiative-equilibrium state with $\Delta_{h}=120 \mathrm{~K}$. Figures 12 and 13 show Hadley circulation strength and extent for

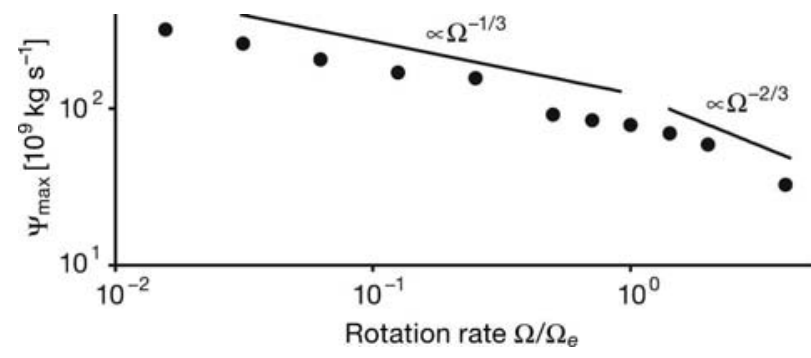

FIG. 10. Hadley circulation strength $\Psi_{\max }$ as a function of rotation rate (relative to Earth's). In all cases, $\Delta_{h}=120 \mathrm{~K}, \gamma=0.7$, and the thermal relaxation time varies spatially. The lines show power laws with exponents $-1 / 3$ and $-2 / 3$. 


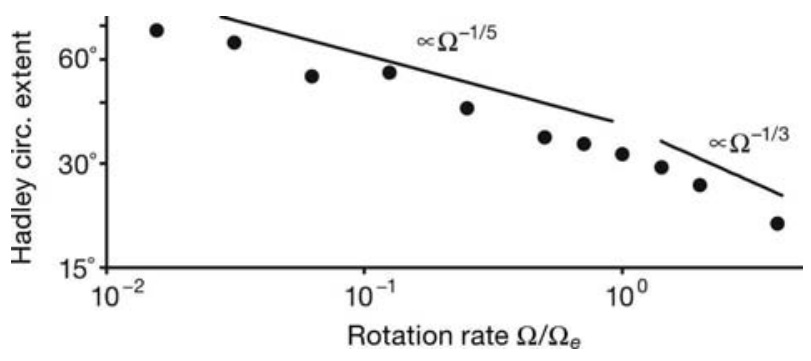

FIG. 11. Hadley circulation extent $\phi_{H}$ as a function of rotation rate (relative to Earth's) for the same simulations as in Fig. 10. The lines show power laws with exponents $-1 / 5$ and $-1 / 3$.

the two modified radiative-equilibrium states and for the normative state from which the modified states are derived.

With flat extratropical radiative-equilibrium temperatures, simulations with $H_{t}^{\prime} \Delta_{h}^{\prime} \leq 0.15\left(\Delta_{h} \leq 45 \mathrm{~K}\right)$ have relatively weak Hadley circulations $\left(\Psi_{\max } \leqslant 3.5 \times\right.$ $10^{9} \mathrm{~kg} \mathrm{~s}^{-1}$ ), with the strength increasing modestly with $H_{t}^{\prime} \Delta_{h}^{\prime}$ (Fig. 12). The four simulations with the smallest temperature contrasts $\left(\Delta_{h}=15,22.5,30\right.$, and $\left.45 \mathrm{~K}\right)$ have little eddy activity, as the temperature gradient in dynamical equilibrium is too small to give rise to a strong baroclinically unstable jet. However, as $H_{t}^{\prime} \Delta_{h}^{\prime}$ increases from 0.15 to $0.21\left(\Delta_{h}=60 \mathrm{~K}\right)$, the subtropical temperature gradient becomes sufficiently large that stronger eddies develop, leading to a jump in Hadley circulation strength $\left(\Psi_{\max } \approx 15 \times 10^{9} \mathrm{~kg} \mathrm{~s}^{-1}\right)$.

At higher $H_{t}^{\prime} \Delta_{h}^{\prime}$, the Hadley circulations in the three series of simulations exhibit similar scaling behavior and are of similar strength: the normative Hadley circulations are about a factor 1.7 stronger than the circulations with flat tropical radiative-equilibrium temperatures and about a factor 1.3 stronger than the circulations with flat extratropical radiative-equilibrium temperatures. There are, however, structural differences between the circulations. In simulations with flat

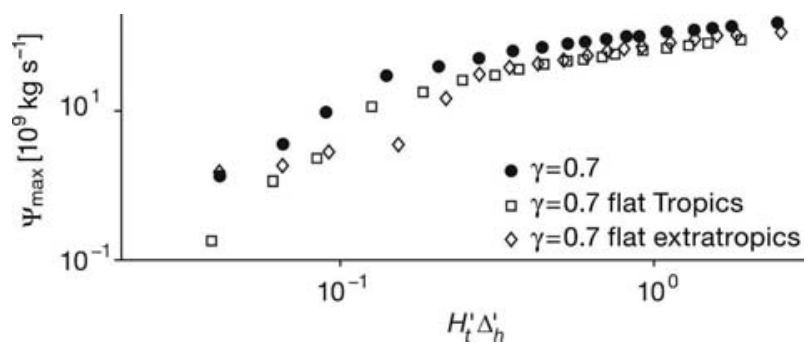

FIG. 12. Hadley circulation strength $\Psi_{\max }$ as a function of $H_{t}^{\prime} \Delta_{h}^{\prime}$ for simulations with normative $\cos ^{2} \phi$ radiative-equilibrium temperatures (filled circles), with flat tropical radiative-equilibrium temperatures (open squares), and with flat extratropical radiativeequilibrium temperatures (open diamonds). In all cases, $\gamma=0.7$.

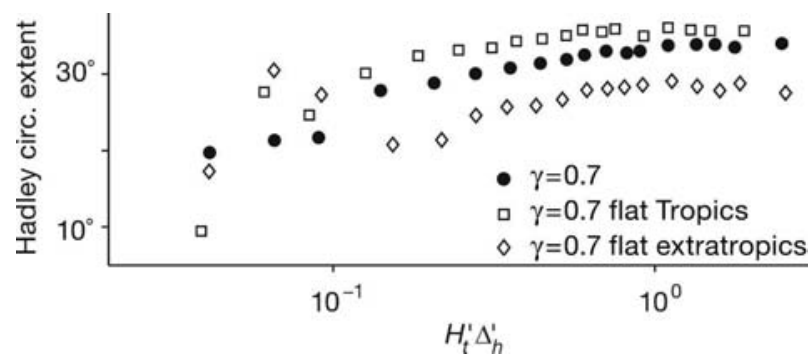

FIG. 13. Hadley circulation extent $\phi_{H}$ as a function of $H_{t}^{\prime} \Delta_{h}^{\prime}$. Symbols have the same meaning as in Fig. 12.

extratropical radiative-equilibrium temperatures, the dynamical-equilibrium temperature gradients are confined almost exclusively to the subtropics, so that the subtropical jet is narrower and typically equatorward of and stronger than that in the corresponding normative simulations. Figure 13 shows that the Hadley circulations are also narrower in these simulations, extending approximately to the equatorward flank of the narrower jet. Conversely, the Hadley circulations in the simulations with flat tropical radiative-equilibrium temperatures are slightly wider than those in the normative simulations, and the subtropical jet is typically weaker. This contrasts with the prediction of HH's nearly inviscid axisymmetric theory, according to which the Hadley circulation narrows as tropical radiative-equilibrium temperature gradients are reduced [cf. Eq. (2a)]. The simulations with flat tropical radiative-equilibrium temperatures generally exhibit smaller local Rossby numbers in the upper branches of the Hadley circulations near the latitudes of the streamfunction extremum $(0.1$ $\lesssim$ Ro $\lesssim 0.4)$ than the normative simulations or the simulations with flat extratropical radiative-equilibrium temperatures $(0.2 \lesssim$ Ro $\lesssim 0.8)$.

With flat tropical temperatures, with $H_{t}^{\prime} \Delta_{h}^{\prime} \lesssim 0.53$ $\left(\Delta_{h} \lesssim 135 \mathrm{~K}\right)$, and with constant thermal relaxation time, nearly inviscid axisymmetric theory would predict the atmosphere to be in radiative-convective equilibrium. The zonal wind in gradient balance with the radiative-convective equilibrium state would not violate the constraint (Hide's theorem) that there must not be an extremum of angular momentum away from the surface (cf. Plumb and Hou 1992). With the spatially varying thermal relaxation time in our simulations, even when the radiative-equilibrium temperature gradient is zero $\left(\Delta_{h}=0\right)$, radiative-convective equilibrium temperatures have a maximum at the equator, with nonzero second derivatives, so nearly inviscid theory predicts there to be a Hadley circulation. However, the Hadley circulation that results in a simulation with $\Delta_{h}=0$ is narrow $\left(\phi_{H} \approx 12^{\circ}\right)$ and weak $\left(\Psi_{\max } \approx 0.1 \times\right.$ $10^{9} \mathrm{~kg} \mathrm{~s}^{-1}$ ) -more than an order of magnitude weaker 
than the Hadley circulations in the simulations with flat tropical radiative-equilibrium temperatures with $\Delta_{h}^{\prime} H_{t}^{\prime} \gtrsim 0.06\left(\Delta_{h} \gtrsim 22.5 \mathrm{~K}\right) .^{5}$ This suggests that eddy dynamics are primarily responsible for the existence of the Hadley circulation in these simulations. Consistent with this interpretation, the Hadley circulation strength in these simulations is similar to $\mathcal{T}$, and the local Rossby number in the upper branches of the circulations is less than about 0.4 .

The simulations with orthogonal complements of tropical and extratropical radiative-equilibrium temperature gradients show clearly that Hadley circulation dynamics, at least in our simulations, cannot be understood independently of eddy dynamics. The Hadley circulation contributes to the maintenance of the mean state on which eddy dynamics are superimposed; conversely, eddy dynamics contribute to the maintenance of the Hadley circulation. The result that, for sufficiently large fixed temperature contrasts $\Delta_{h}$, the strength scaling of the Hadley circulation remains essentially unaffected if either tropical or extratropical radiative-equilibrium temperature gradients are eliminated calls into question the degree to which changes in Hadley circulation strength can be viewed, as is sometimes assumed, as controlled by tropical processes. As shown in Figs. 12 and 13, the Hadley circulation also responds to perturbations in extratropical radiativeequilibrium temperature gradients even when the tropical radiative-equilibrium temperature gradients are held fixed.

\section{Simulations with an off-equatorial heating maximum}

In Walker and Schneider (2005), we investigated the response of the Hadley circulation to displacing the maximum radiative-equilibrium temperature off of the equator, focusing on the question of whether the annually averaged Hadley circulation is nonlinearly amplified compared with the Hadley circulation driven by

\footnotetext{
${ }^{5}$ Consistent with the prediction of nearly inviscid axisymmetric theory and unlike in the simulations described by Kirtman and Schneider (2000), the simulated atmosphere in our model appears to converge slowly to a state of radiative-convective equilibrium without a large-scale circulation if temperature gradients in radiative-convective equilibrium are eliminated by setting $\Delta_{h}=0$ and using a constant thermal relaxation time. (We stopped the integration after 2100 simulated days, at which time there still was a very weak circulation with $\Psi_{\max } \approx 10^{4} \mathrm{~kg} \mathrm{~s}^{-1}$.) So there is no evidence in our simulations for mechanisms of maintaining a large-scale Hadley circulation in the absence of radiativeconvective equilibrium temperature gradients (cf. Kirtman and Schneider 2000).
}

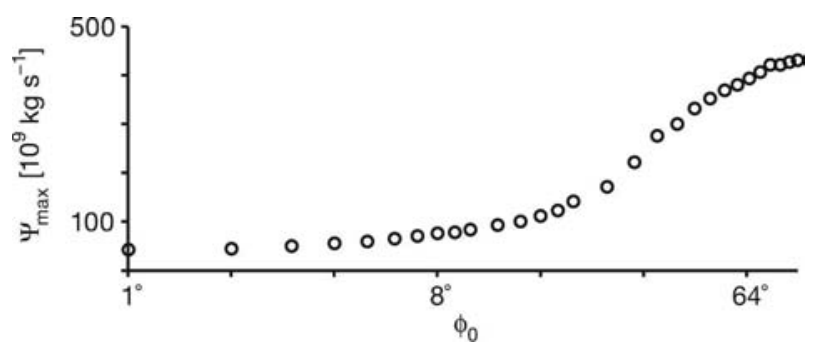

FIG. 14. Strength $\Psi_{\max }$ of cross-equatorial Hadley cell as a function of $\phi_{0}$ in simulations with constant thermal relaxation time. (The scale of the $\phi_{0}$ axis is logarithmic.) In all cases, $\gamma=0.7$ and $\Delta_{h}=120 \mathrm{~K}$.

relaxation toward annually averaged radiative-equilibrium temperatures. We considered a $6^{\circ}$ displacement of the maximum radiative-equilibrium temperature off the equator and did not attempt to find a functional relation between Hadley circulation strength and the latitude $\phi_{0}$ of maximum radiative-equilibrium temperature. In an extension of this earlier study, Fig. 14 shows the strength of the cross-equatorial Hadley cell (again understood as the maximum absolute value of the mass flux streamfunction above the level $\sigma=0.7$ ) in simulations in which the latitude $\phi_{0}$ of maximum radiativeequilibrium temperature (3) takes values from $1^{\circ}$ to $90^{\circ}$. To facilitate comparisons with nearly inviscid axisymmetric theories, the thermal relaxation time in these simulations is constant and equal to 50 days throughout the atmosphere.

The scaling of the strength as a function of $\phi_{0}$ is in three regimes: for $\phi_{0} \lesssim 16^{\circ}$, the strength increases weakly with $\phi_{0}$ (roughly as $\phi_{0}^{1 / 3}$ or more slowly); for $16^{\circ} \lesssim \phi_{0} \lesssim 50^{\circ}$, the strength increases more strongly with $\phi_{0}$ (roughly as $\phi_{0}^{4 / 3}$ ); and for $\phi_{0} \gtrsim 50^{\circ}$, the strength appears to saturate and increase slowly with $\phi_{0}$. We have obtained similar scaling behavior with regime transitions in simulations in which the thermal relaxation time near the surface varied spatially, with shorter relaxation times near the maximum in radiativeequilibrium surface temperature. The latitudes $\phi_{0}$ at which the regime transitions occurred were smaller than in the simulations with constant thermal relaxation time shown in Fig. 14, and saturation of the strength for large $\phi_{0}$ was more pronounced, but qualitatively the results were similar.

The nearly inviscid axisymmetric theory of Lindzen and Hou (1988) does not account for the transition in scaling regimes near $\phi_{0}=16^{\circ}$. By numerical calculations analogous to those of Lindzen and Hou but with the radiative-convective equilibrium state of our simulations, we have verified that the nearly inviscid axisymmetric theory for our simulations leads to an approximate power-law dependence of strength on $\phi_{0}$ for 
$\phi_{0} \lesssim 50^{\circ}$ (Fig. 14). The strength saturates for $\phi_{0} \geqslant 50^{\circ}$, similar to what is seen in the simulations. The strength increases roughly as $\phi_{0}^{4 / 3}$ for all $\phi_{0} \lesssim 50^{\circ}$, similar to what is seen in the simulations for $16^{\circ} \lesssim \phi_{0} \lesssim 50^{\circ}$. The nearly inviscid axisymmetric theory does not predict a regime transition for $\phi_{0} \lesssim 50^{\circ}$, and the predicted structure of the circulations also differs from those seen in the simulations. For example, according to the nearly inviscid axisymmetric theory, the streamfunction maximum of the cross-equatorial Hadley cell moves poleward in the southern (winter) hemisphere with increasing $\phi_{0}$ (cf. Walker and Schneider 2005), whereas the streamfunction maximum in our simulations moves equatorward with increasing $\phi_{0} \lesssim 16^{\circ}$ and stays near the equator in the northern (summer) hemisphere as $\phi_{0}$ is increased further.

In the simulations, the transition in scaling regimes near $\phi_{0}=16^{\circ}$ occurs when the streamfunction maximum moves across the equator: simulations with $\phi_{0} \leq$ $16^{\circ}$ have a streamfunction maximum in the winter hemisphere; simulations with $\phi_{0}>16^{\circ}$ have a streamfunction maximum in the summer hemisphere (see Fig. 15 for examples). When the streamfunction maximum is in the winter hemisphere, it is of similar magnitude as the quantity $\mathcal{T}$, related to the eddy momentum flux divergence at the latitude of the streamfunction maximum. The local Rossby number in the upper branch of the cross-equatorial Hadley cell, at the latitude of the streamfunction maximum, increases from $\mathrm{Ro} \approx 0.3$ for $\phi_{0}=2^{\circ}$ to $\operatorname{Ro} \approx 0.6$ for $\phi=14^{\circ}$, indicating that nonlinear momentum advection by the Hadley cell becomes increasingly more important as $\phi_{0}$ and the strength of the Hadley cell increase, but the upper-level flow remains relatively far from angular momentumconserving. In contrast, when the streamfunction maximum is in the summer hemisphere, it is located within $5^{\circ}$ of the equator, shielded from midlatitude eddies in the winter hemisphere by the zero-wind line. The crossequatorial Hadley cells in the $\phi_{0}>16^{\circ}$ simulations are therefore closer to angular momentum-conserving, with the ascending branch closely following angular momentum contours (see Fig. 15c for an example). The descending branch is located in the winter subtropics, where eddy momentum flux divergence makes it possible that streamlines cross angular momentum contours.

This transition in scaling regimes is similar to the transition in scaling regimes of axisymmetric circulations from a linear viscous regime to a nonlinear, nearly angular momentum-conserving regime described by Plumb and Hou (1992), but it occurs for different reasons. The transition discussed by Plumb and Hou, in response to increasing subtropical thermal driving, oc-
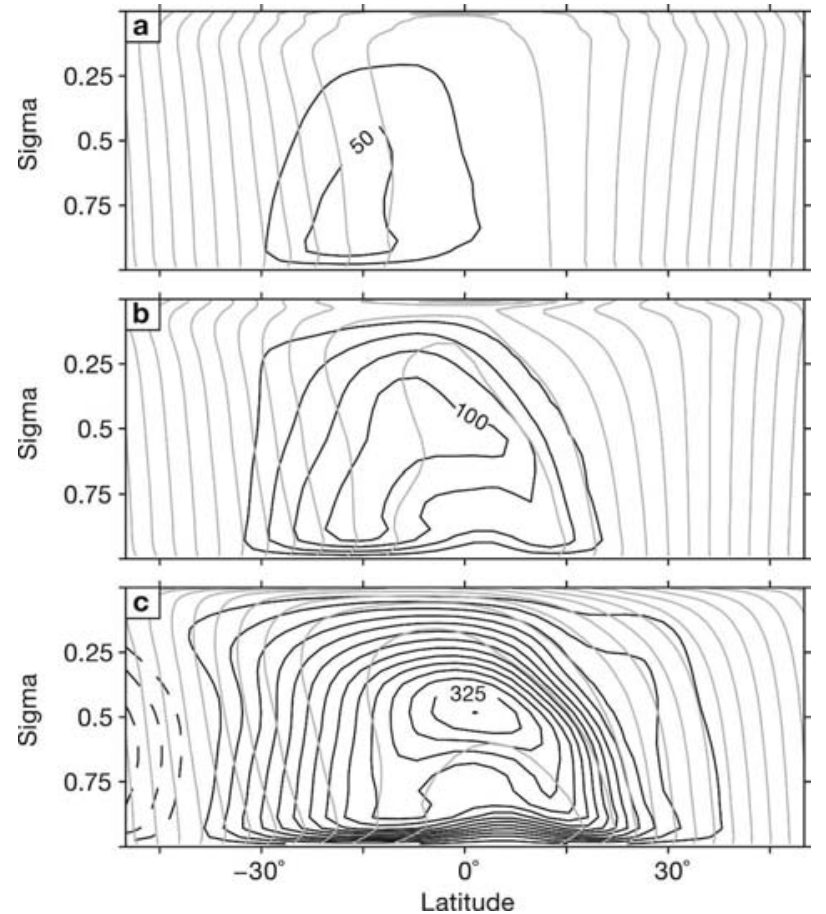

FIG. 15. Mass flux streamfunction (black contours) and angular momentum (gray contours) for (a) $\phi_{0}=4^{\circ}$, (b) $\phi_{0}=16^{\circ}$, and (c) $\phi_{0}=50^{\circ}$. Contour intervals are $2.5 \times 10^{9} \mathrm{~kg} \mathrm{~s}^{-1}$ and $0.05 \Omega a^{2}$ for streamfunction and angular momentum. In all cases, $\gamma=0.7$ and $\Delta_{h}=120 \mathrm{~K}$.

curs when the zonal wind in gradient balance with the radiative-convective equilibrium state violates Hide's constraint that there must not be an extremum of angular momentum away from the surface, making a nonlinear circulation necessary. In particular, the transition only occurs if the radiative-convective equilibrium temperature gradients at the equator vanish. In contrast, the transition in our simulations, with nonzero radiative-convective equilibrium temperature gradients at the equator, is from a regime in which eddy momentum fluxes strongly influence the strength of the Hadley circulation to a regime in which the influence of eddy momentum fluxes is weak and hence the circulation is closer to angular momentum-conserving because eddies cannot reach the latitude of the streamfunction extremum.

Hadley circulations for large $\phi_{0}$ (see Fig. $15 \mathrm{c}$ for an example) resemble the circulation of Mars during northern solstice in that they have a near-surface westerly jet in the summer hemisphere that extends from the Tropics to midlatitudes and a strong meridional surface pressure gradient associated with near-surface temperature gradients, with pressure decreasing from the winter hemisphere midlatitudes to the summer hemisphere midlatitudes [compare, e.g., Haberle et al. 
(1993); see Schneider (1983) for a discussion of related axisymmetric circulations]. For $\phi_{0}=60^{\circ}$, for example, the zonal wind has a maximum of $25 \mathrm{~m} \mathrm{~s}^{-1}$ near $22^{\circ}$ at a pressure of about $900 \mathrm{hPa}$. The surface pressure has a global maximum of $1043 \mathrm{hPa}$ near $-45^{\circ}$ and decreases monotonically to a local minimum of $967 \mathrm{hPa}$ near 55 maintaining the near-surface return flow of the crossequatorial Hadley cell against surface drag. The structure of the cross-equatorial Hadley cells-for example, the latitudes of strongest ascent and descent-changes little as $\phi_{0}$ is increased beyond the value $\phi_{0}=50^{\circ}$ for which the streamfunction and angular momentum contours are shown in Fig. 15c. In particular, the latitude of the main ascent region does not move significantly further poleward as $\phi_{0}$ is increased further. (In contrast, according to the nearly inviscid axisymmetric theory of Lindzen and Hou, the latitude of the ascent region continues to move poleward as $\phi_{0}$ increases beyond $\phi_{0}=$ $50^{\circ}$. However, one assumption of the theory-that the near-surface winds are weak - is violated in these simulations.) Some near-surface mass flux streamlines have a horseshoe shape in low latitudes, with ascent on the winter side and descent on the summer side of the streamfunction maximum, apparently for the reasons discussed by Pauluis (2004). Streamlines of the circulations for large $\phi_{0}$ nearly coincide with angular momentum surfaces in the ascending and upper branches (Fig. $15 \mathrm{c})$, suggesting that axisymmetric theories that, for example, couple a nearly inviscid free troposphere to a dissipative boundary layer (cf. Pauluis 2004) may account for some aspects of these circulations. However, eddy momentum flux divergences are still significant in the broad descending branches of the circulations.

\section{Conclusions}

By varying the rotation rate, radius, and thermal driving in an idealized GCM, we have shown that in our model, the strength and meridional extent of the Hadley circulation display scaling behavior inconsistent with existing theories. We have also shown that, over a wide range of flow parameters, albeit not always, the strength of the Hadley circulation is linearly related to the eddy momentum flux divergence and that it is this relation that leads to disagreement between the scaling behavior exhibited by our simulations and that predicted by nonlinear nearly inviscid theories of axisymmetric Hadley circulations. The linear relation between Hadley circulation strength and eddy momentum flux divergence is more consistent with linear theories of axisymmetric Hadley circulations, but these are likewise not universally adequate. As the heating is progressively displaced further away from the equator, the
Hadley circulation undergoes a transition to a regime with a nearly angular momentum-conserving upper branch, a regime in which nonlinear theories of axisymmetric Hadley circulations may be more adequate.

Aspects of the scaling behavior of the Hadley circulation extent, such as the increase of the extent with increasing tropical static stability, are consistent with the notion that baroclinic instability limits the extent of the Hadley circulation. Similar mechanisms, in response to a decreased moist adiabatic lapse rate and thus increased tropical (dry) static stability in a warmer climate, may be responsible for the poleward expansion of the Hadley circulation and the poleward shift of storm tracks seen in simulations of climates with increased concentrations of greenhouse gases (e.g., Yin 2005).

Our simulations with hemispherically symmetric thermal driving span circulations with local Rossby numbers in the upper branch of the Hadley circulation, at the latitude of the streamfunction extremum, between 0.1 and 0.8. This indicates that neither nonlinear nearly inviscid theories, valid for Ro $\rightarrow 1$, nor linear theories, valid for Ro $\rightarrow 0$, of axisymmetric Hadley circulations can be expected to be generally adequate. To understand the scaling behavior of Hadley circulations in which nonlinear momentum fluxes associated with both the mean flow and eddies may be significant, we need a new Hadley circulation theory that can account for circulations with intermediate Rossby numbers. The weak temperature gradient approximation of Sobel et al. (2001) may provide a framework within which a theory for Hadley circulations with intermediate Rossby numbers can be developed (Polvani and Sobel 2002). Eddy dynamics would appear as forcings and boundary conditions in such a theory and, to obtain a closed theory, must be related to the mean atmospheric state that is, in part, determined by the Hadley circulation itself (Schneider 2006).

We have focused on eddy momentum fluxes and their influence on the Hadley circulation. Eddy heat fluxes likewise influence the Hadley circulation, for example, in that the Hadley circulation is constrained by the requirement that diabatic heating in the Tropics balance cooling in the subtropics by both radiative processes and eddy heat export to the extratropics. However, eddy heat fluxes can be incorporated into existing Hadley circulation theories, for example, in theories using Newtonian relaxation to represent diabatic processes, by changing the radiative-equilibrium temperature to which temperatures are relaxed (cf. Schneider 1984).

The importance of eddy fluxes of heat and momentum in the dynamics of the Hadley circulation has 
broad implications for the general circulation. The baroclinic eddies that effect the eddy fluxes are directly related to extratropical circulation characteristics such as meridional temperature gradients, suggesting that tropical circulation characteristics may depend on the extratropical climate.

Acknowledgments. We thank Dorian Abbot, Richard Lindzen, Edwin Schneider, Adam Sobel, and an anonymous reviewer for very helpful comments on an earlier version of this paper. We are grateful for financial support by the Davidow Discovery Fund, by an Alfred P. Sloan Research Fellowship, and by the National Science Foundation (Grant ATM-0450059) and for computing resources provided by the National Center for Atmospheric Research (which is sponsored by the National Science Foundation). The program code for the simulations described in this paper, as well as the simulation results themselves, are available from the authors upon request.

\section{REFERENCES}

Becker, E., G. Schmitz, and R. Geprägs, 1997: The feedback of midlatitude waves onto the Hadley cell in a simple general circulation model. Tellus, 49A, 182-199.

Bourke, W., 1974: A multi-level spectral model. I. Formulation and hemispheric integration. Mon. Wea. Rev., 102, 687-701.

Dickinson, R. E., 1971: Analytic model for zonal winds in the Tropics. I. Details of the model and simulation of gross features of the zonal mean troposphere. Mon. Wea. Rev., 99, $501-510$.

Dima, I. M., and J. M. Wallace, 2003: On the seasonality of the Hadley cell. J. Atmos. Sci., 60, 1522-1527.

Haberle, R. M., J. B. Pollack, J. R. Barnes, R. W. Zurek, C. B. Leovy, J. R. Murphy, H. Lee, and J. Schaeffer, 1993: Mars atmospheric dynamics as simulated by the NASA Ames general circulation model. 1. The zonal-mean circulation. J. Geophys. Res., 98, 3093-3123.

Held, I. M., cited 2000: The general circulation of the atmosphere. Proc. Program in Geophysical Fluid Dynamics, Woods Hole, MA, Woods Hole Oceanographic Institution. [Available online at www.gfd.whoi.edu/proceedings/2000/ PDFvol2000.html.]

, and A. Y. Hou, 1980: Nonlinear axially symmetric circulations in a nearly inviscid atmosphere. J. Atmos. Sci., 37, 515533.

— - and T. Schneider, 1999: The surface branch of the zonally averaged mass transport circulation in the troposphere. $J$. Atmos. Sci., 56, 1688-1697.

Hide, R., 1969: Dynamics of the atmospheres of the major planets with an appendix on the viscous boundary layer at the rigid bounding surface of an electrically-conducting rotating fluid in the presence of a magnetic field. J. Atmos. Sci., 26, 841853.

Kållberg, P., A. Simmons, S. Uppala, and M. Fuentes, 2004: The ERA-40 archive. ECMWF Tech. Rep., European Centre for Medium-Range Weather Forecasts, 31 pp. [Available online at www.ecmwf.int/publications.]
Kim, H.-K., and S. Lee, 2001: Hadley cell dynamics in a primitive equation model. Part II: Nonaxisymmetric flow. J. Atmos. Sci., 58, 2859-2871.

Kirtman, B. P., and E. K. Schneider, 2000: A spontaneously generated tropical atmospheric general circulation. J. Atmos. Sci., 57, 2080-2093.

Koh, T.-Y., and R. A. Plumb, 2004: Isentropic zonal average formulation and the near-surface circulation. Quart. J. Roy. Meteor. Soc., 130, 1631-1654.

Lindzen, R.-S., and A. Y. Hou, 1988: Hadley circulations for zonally averaged heating centered off the equator. J. Atmos. Sci., 45, 2416-2427.

Navarra, A., and G. Boccaletti, 2002: Numerical general circulation experiments of sensitivity to Earth rotation rate. Climate Dyn., 19, 467-483.

Pauluis, O., 2004: Boundary layer dynamics and cross-equatorial Hadley circulation. J. Atmos. Sci., 61, 1161-1173.

Plumb, R. A., and A. Y. Hou, 1992: The response of a zonally symmetric atmosphere to subtropical thermal forcing: Threshold behavior. J. Atmos. Sci., 49, 1790-1799.

Polvani, L. M., and A. H. Sobel, 2002: The Hadley circulation and the weak temperature gradient approximation. J. Atmos. Sci., 59, 1744-1752.

Schneider, E. K., 1977: Axially symmetric steady-state models of the basic state for instability and climate studies. Part II. Nonlinear calculations. J. Atmos. Sci., 34, 280-296.

_, 1983: Martian great dust storms: Interpretive axially symmetric models. Icarus, 55, 302-331.

_- 1984: Response of the annual and zonal mean winds and temperatures to variations in the heat and momentum sources. J. Atmos. Sci., 41, 1093-1115.

— , and R. S. Lindzen, 1976: The influence of stable stratification on the thermally driven tropical boundary layer. $J$. Atmos. Sci., 33, 1301-1307.

_ the basic state for instability and climate studies. Part I. Linearized calculations. J. Atmos. Sci., 34, 263-279.

Schneider, T., 2004: The tropopause and the thermal stratification in the extratropics of a dry atmosphere. J. Atmos. Sci., 61, 1317-1340.

_ 2005: Zonal momentum balance, potential vorticity dynamics, and mass fluxes on near-surface isentropes. J. Atmos. Sci., 62, 1884-1900.

- 2006: The general circulation of the atmosphere. Annu. Rev. Earth Planet. Sci., 34, 655-688.

—, and C. C. Walker, 2006: Self-organization of atmospheric macroturbulence into critical states of weak nonlinear eddyeddy interactions. J. Atmos. Sci., 63, 1569-1586.

Sobel, A. H., J. Nilsson, and L. M. Polvani, 2001: The weak temperature gradient approximation and balanced tropical moisture waves. J. Atmos. Sci., 58, 3650-3665.

Uppala, S. M., and Coauthors, 2005: The ERA-40 re-analysis. Quart. J. Roy. Meteor. Soc., 131, 2961-3012.

Walker, C. C., and T. Schneider, 2005: Response of idealized Hadley circulations to seasonally varying heating. Geophys. Res. Lett., 32, L06813, doi:10.1029/2004GL022304.

Williams, G. P., 1988a: The dynamical range of global circulations- I. Climate Dyn., 2, 205-260.

— 1988b: The dynamical range of global circulations-II. Climate Dyn., 3, 45-84.

Yin, J. H., 2005: A consistent poleward shift of the storm tracks in simulations of 21st century climate. Geophys. Res. Lett., 32, L18701, doi:10.1029/2005GL023684. 